\title{
Separate But Interactive Parallel Olfactory Processing Streams Governed by Different Types of GABAergic Feedback Neurons in the Mushroom Body of a Basal Insect
}

\author{
Naomi Takahashi, ${ }^{1}$ Hiroshi Nishino, ${ }^{2}$ Mana Domae, ${ }^{2}$ and ${ }^{\circledR}$ Makoto Mizunami ${ }^{3}$ \\ ${ }^{1}$ Graduate School of Life Science, Hokkaido University, Sapporo 060-0810, Japan, ${ }^{2}$ Research Institute for Electronic Science, Hokkaido University, Sapporo \\ 060-0812, Japan, and 3Faculty of Science, Hokkaido University, Sapporo 060-0810, Japan
}

The basic organization of the olfactory system has been the subject of extensive studies in vertebrates and invertebrates. In many animals, GABA-ergic neurons inhibit spike activities of higher-order olfactory neurons and help sparsening of their odor representations. In the cockroach, two different types of GABA-immunoreactive interneurons (calyceal giants [CGs]) mainly project to the base and lip regions of the calyces (input areas) of the mushroom body (MB), a second-order olfactory center. The base and lip regions receive axon terminals of two different types of projection neurons, which receive synapses from different classes of olfactory sensory neurons (OSNs), and receive dendrites of different classes of Kenyon cells, $\mathrm{MB}$ intrinsic neurons. We performed intracellular recordings from pairs of CGs and MB output neurons (MBONs) of male American cockroaches, the latter receiving synapses from Kenyon cells, and we found that a CG receives excitatory synapses from an MBON and that odor responses of the MBON are changed by current injection into the CG. Such feedback effects, however, were often weak or absent in pairs of neurons that belong to different streams, suggesting parallel organization of the recurrent pathways, although interactions between different streams were also evident. Cross-covariance analysis of the spike activities of CGs and MBONs suggested that odor stimulation produces synchronized spike activities in MBONs and then in CGs. We suggest that there are separate but interactive parallel streams to process odors detected by different OSNs throughout the olfactory processing system in cockroaches.

Key words: GABA; insect; mushroom body; olfaction; parallel processing; synchronization

Significance Statement

Organizational principles of the olfactory system have been the subject of extensive studies. In cockroaches, signals from olfactory sensory neurons $(\mathrm{OSN})$ in two different classes of sensilla are sent to two different classes of projection neurons, which terminate in different areas of the mushroom body (MB), each area having dendrites of different classes of MB intrinsic neurons (Kenyon cells) and terminations of different classes of GABAergic neurons. Physiological and morphological assessments derived from simultaneous intracellular recordings/stainings from GABAergic neurons and MB output neurons suggested that GABAergic neurons play feedback roles and that odors detected by OSNs are processed in separate but interactive processing streams throughout the central olfactory system.

\section{Introduction}

Animals are required to process complex and dynamic olfactory stimuli for orientation, communication, and predator avoidance.

Received Jan. 11, 2019; revised Sept. 8, 2019; accepted Sept. 17, 2019.

Author contributions: N.T. and M.M. designed research; N.T. and M.D. performed research; N.T. analyzed data; N.T. wrote the first draft of the paper; N.T., H.N., and M.M. edited the paper; N.T., H.N., and M.M. wrote the paper.

This work was supported by Japan Science Society Sasakawa Scientific Research Grant 29-438 to N.T., Japan Society for the Promotion of Science Overseas Challenge Program for Young Researchers 201780130 to N.T., and Ministry of Education, Science, Culture, Sports and Technology of Japan Grants-in-Aid for Scientific Research 17 K07479 to H.N. and 16H04814, 16K18586, and 19H03261 to M.M. We thank Kayoko Suenaga (Carl Zeiss Microscopy) for help with observation by LSM 880 equipped with Airyscan Fast and 3D reconstruction by Imaris; and Drs. Kentaro Arikawa and Atsuko Matsushita (Sokendai) for help with observations of neural morphology.
Organizational principles of the olfactory system to achieve these goals have been extensively explored in vertebrates and invertebrates. Major issues of debate include the parallel nature of the olfactory system (Galizia and Rössler, 2010) and mechanisms of the formation of sparse odor representation in higher olfactory centers (Perez-Orive et al., 2004). These issues have been exam-

The authors declare no competing financial interests.

Correspondence should be addressed to Makoto Mizunami at mizunami@sci.hokudai.ac.jp.

N. Takahashi's present address: Department of Biology, Philipps-University, Marburg 35032, Germany.

https://doi.org/10.1523/JNEUROSCI.0088-19.2019

Copyright $\odot 2019$ the authors 
ined in this study by using nocturnal cockroaches as model animals, which heavily rely on communications using olfaction.

In the olfactory systems of insects, olfactory sensory neurons (OSNs) and second-order neurons called projection neurons (PNs) typically respond to a variety of odors with a burst of spikes. In contrast, third-order neurons (Kenyon cells [KCs]) in a higher-order center (the mushroom body [MB]), which play critical roles in olfactory learning (Menzel and Giurfa, 2006; Liu et al., 2012), respond only to specific odors with a few spikes, achieving sparse odor coding (Gupta and Stopfer, 2014; Lin et al., 2014). One mechanism contributing to the formation of spatially and temporally sparse odor representations in populations of KCs is widespread and broadly tuned GABA-ergic inhibition that feeds signals of KCs back to KCs (Szyszka et al., 2005; Papadopoulou et al., 2011). This type of global inhibition lessens odorevoked excitatory responses of individual KCs so that only cells receiving strong and preferred excitation are driven to spike. Axons of KCs project to the lobes and make synapses with $\mathrm{MB}$ output neurons (MBONs) that respond to a variety of odors with bursts of spikes. MBONs participate in the control of behavioral responses to learned odors (Plaçais et al., 2013).

The cockroach Periplaneta americana possesses four tightly bundled GABA-immunoreactive neurons, termed calyceal giants (CGs) (Yamazaki et al., 1998), in each hemisphere of the brain (Fig. 1B). CGs have terminal branches in the calyces (input area) of the MB. Dendritic branches of CGs are distributed in the protocerebrum in close proximity to terminal branches of some MBONs, and it has thus been deduced that CGs receive signals from $\mathrm{MBONs}$ and serve as feedback roles (Nishino and Mizunami, 1998; Strausfeld and Li, 1999a; Takahashi et al., 2017). This is in contrast to the fact that GABAimmunoreactive feedback neurons of the $\mathrm{MB}$ in other species feed signals of KCs back to KCs (Stopfer, 2014). However, no evidence has been provided to convincingly demonstrate feedback roles of the CGs.

The four CGs are categorized into Type 1, nonspiking (NS), Type 2a, and Type $2 \mathrm{~b}$ based on their morphology and physiology (Takahashi et al., 2017). The Type 1 CG supplies axon terminals nearly exclusively to the basal region of the $\mathrm{MB}$ calyces, where Type 1 PNs send axon terminals. The remaining three CGs extend terminal arborizations densely in the lip (peripheral) region of the calyces, where Type 2 PNs provide axon terminals, and sparsely in a part of the base region. Type 1 PNs receive synapses from OSNs housed in trichoid and grooved basiconic sensilla on antennae, whereas Type 2 PNs receive synapses from OSNs housed in perforated basiconic sensilla (Watanabe et al., 2017) (Fig. 1A). Moreover, two different morphological classes of KCs, Class I and Class II KCs (Mizunami et al., 1998a,b; Strausfeld and Li, 1999b), extend dendrites in the lip and base regions, respectively (Nishino et al., 2018). Therefore, dual parallel processing is maintained from OSNs to the KCs. A question arises whether such a parallel processing system continues to the feedback circuits of the MB.

To address this question and to investigate the basic organization of neural circuits in MBs, we performed simultaneous intracellular recording from a CG and an MBON in cockroaches. The results suggest that CGs indeed serve as feedback neurons and that odors detected by different types of OSNs are processed in parallel but interactive pathways throughout the central olfactory system in cockroaches, features that have not been reported in any other animals.

\section{Materials and Methods}

\section{Animals}

Adult male cockroaches ( $P$. americana L.) were raised in our laboratory colony under a $12 / 12 \mathrm{~h}$ (light-dark) cycle at $27^{\circ} \mathrm{C}$. Physiological recordings were performed in the subjective night (during the $12 \mathrm{~h}$ dark cycle) since cockroaches are nocturnal animals. A total of 253 cockroaches were used for intracellular recording and staining of CGs and/or MBONs. Among them, simultaneous intracellular recordings were successfully made from a CG (Type 1, NS, or Type 2a) and an MBON in 24 animals (Table 1).

\section{Simultaneous intracellular recording}

The procedures for dissection of animals and intracellular recording were modified from those used in previous studies (Nishino et al., 2003; Takahashi et al., 2017). An anesthetized cockroach was mounted on an experimental chamber with low melting-point wax. Each antenna was immobilized by threading a plastic ring (diameter: $1 \mathrm{~mm}$ ) on the chamber. The cuticle between the two antennae was opened to expose the brain. The brain sheath was softened by Actinase E (Kaken Seiyaku). In the present study, the softened sheath was partly removed mechanically with fine tweezers when we aimed at neurites near the surface of the brain. To stabilize the brain, a rod of platinum ground electrode was inserted into the cavity formed by removal of the esophagus. The brain and the ground electrode were immersed in cockroach saline solution (in mM as follows): $201.2 \mathrm{NaCl}, 3.1 \mathrm{KCl}, 1.8 \mathrm{CaCl}_{2}, 0.2 \mathrm{NaH}_{2} \mathrm{PO}_{4}$, and 1.8 $\mathrm{Na}_{2} \mathrm{HPO}_{4}, \mathrm{pH}$ 7.2. A borosilicate glass microelectrode pulled by a laser puller (P-2000, Sutter Instruments) was filled with $8 \%$ Lucifer yellow (LY, Sigma-Aldrich) in $1 \mathrm{M} \mathrm{LiCl}$ or $10 \%$ micro-Ruby (dextran, tetramethylrhodamine and biotin, $3000 \mathrm{MW}$; Thermo Fisher Scientific) in cockroach saline. Electrodes were inserted into the right hemisphere of the brain unless otherwise stated. Activities of CGs were recorded from the dendrites in the lateral horn (LH), where large EPSPs were visible (Nishino and Mizunami, 1998), or the axonal branches, where EPSPs were small or absent, in the junction where the lateral and medial calyces meet (Takahashi et al., 2017). Activities of MBONs were recorded from the dendrites in the $\mathrm{MB}$ lobes or the axonal branches running mediolaterally in the anterior surface of the brain. Distinctions of electrical activities of MBONs in the dendrites and axon terminals were similar to those found for CGs. Neural activities of individual neurons were amplified with a $30 \mathrm{kHz}$ low-pass filter by an amplifier (Axoclamp 2B, Molecular Devices; and MEZ-8301, Nihon Kohden). Physiological data were stored in a PC with a 10 or $20 \mathrm{kHz}$ sampling rate by an A/D converter and associated software (Micro1401-3 and Spike2 version 6.18, Cambridge Electronic Design).

\section{Odor stimulation}

Odors were delivered to the right antenna by a continuous airflow system described in detail by Nishino et al. (2003). Briefly, compressed air filtered by cotton and charcoal was divided into two flexible Tygon tubes (Saint-Govain). One tube was connected directly to a glass tube $(2 \mathrm{~cm}$ in inner diameter) covering the right antenna. The other tube was further divided by a solenoid valve into one blank tube and four odor-containing tubes, which were connected to the glass tube covering the right antenna. Before odor stimulation, air was passed thorough the blank tube constantly. When the solenoid valve was opened, the air pathway was switched from the blank tube to one of the odor-containing tubes, which was arbitrarily selected with manually operated valves attached to the tubes. An electric signal to open the solenoid valve for $0.5 \mathrm{~s}$ was stored in a PC. A piece of filter paper $(5 \times 40 \mathrm{~mm})$ soaked with odorants was placed in a cartridge of each odor-containing tube. We used monomolecular odorants, 1-hexanol, 1,8-cineol, 1-hexanal, and hexanoic acid (Wako Pure Chemical Industries); OSNs in perforated basiconic sensilla of cockroaches exhibit a high sensitivity to alcohols, whereas those in trichoid and grooved basiconic sensilla exhibit a high sensitivity to fatty acids (Fujimura et al., 1991; Watanabe et al., 2012). The odorants were diluted 100 times by mineral oil. Residual air in the recording cage was continuously sucked out of the room through a vacuum system. The air flow speed in the wind tunnel covering the antenna was adjusted to $20 \mathrm{~cm}$ $\mathrm{s}^{-1}$. The time course of odor delivery was measured by a photo- 
A Olfactory pathways
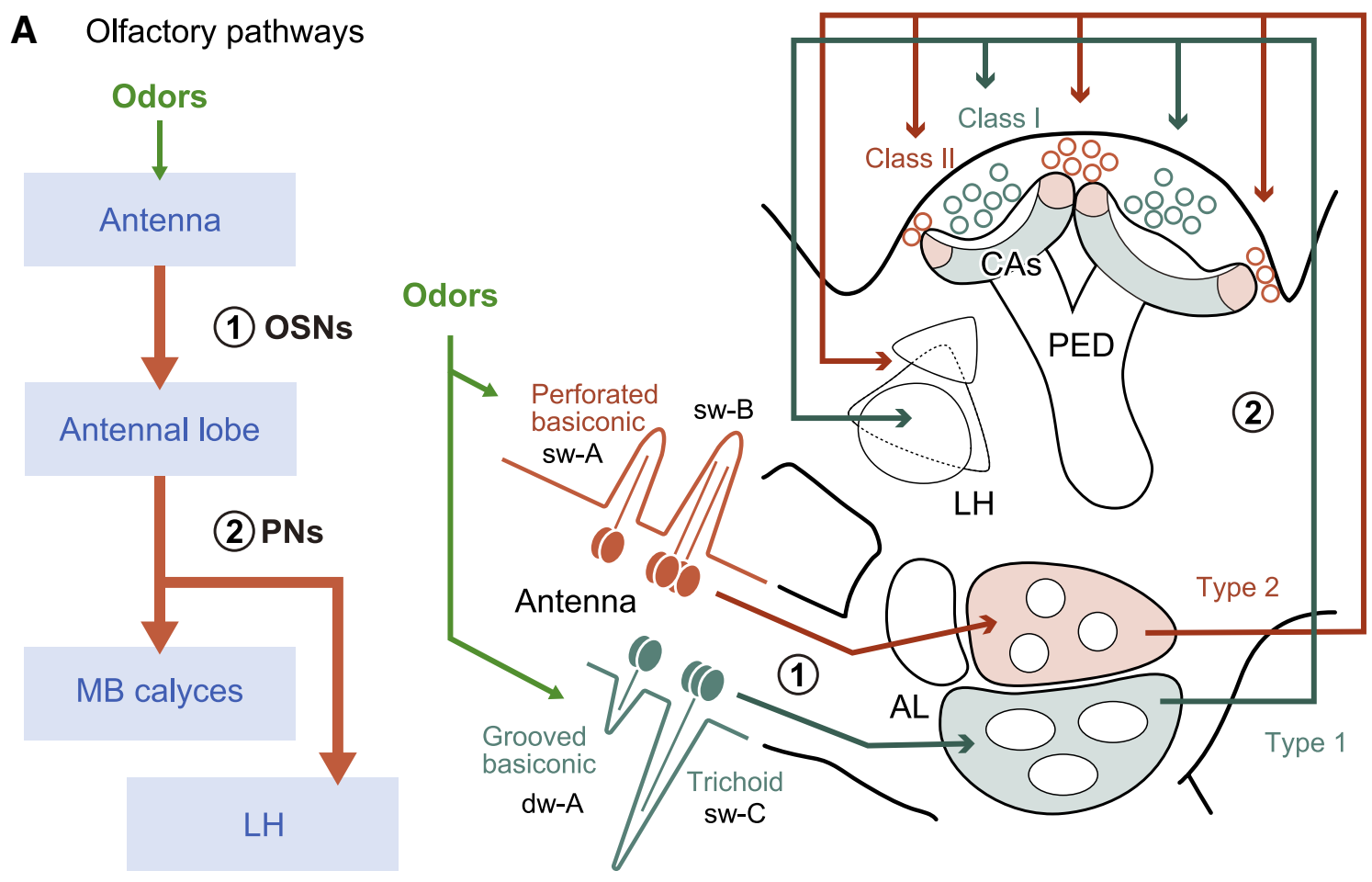

(5)

B MB feedback pathways
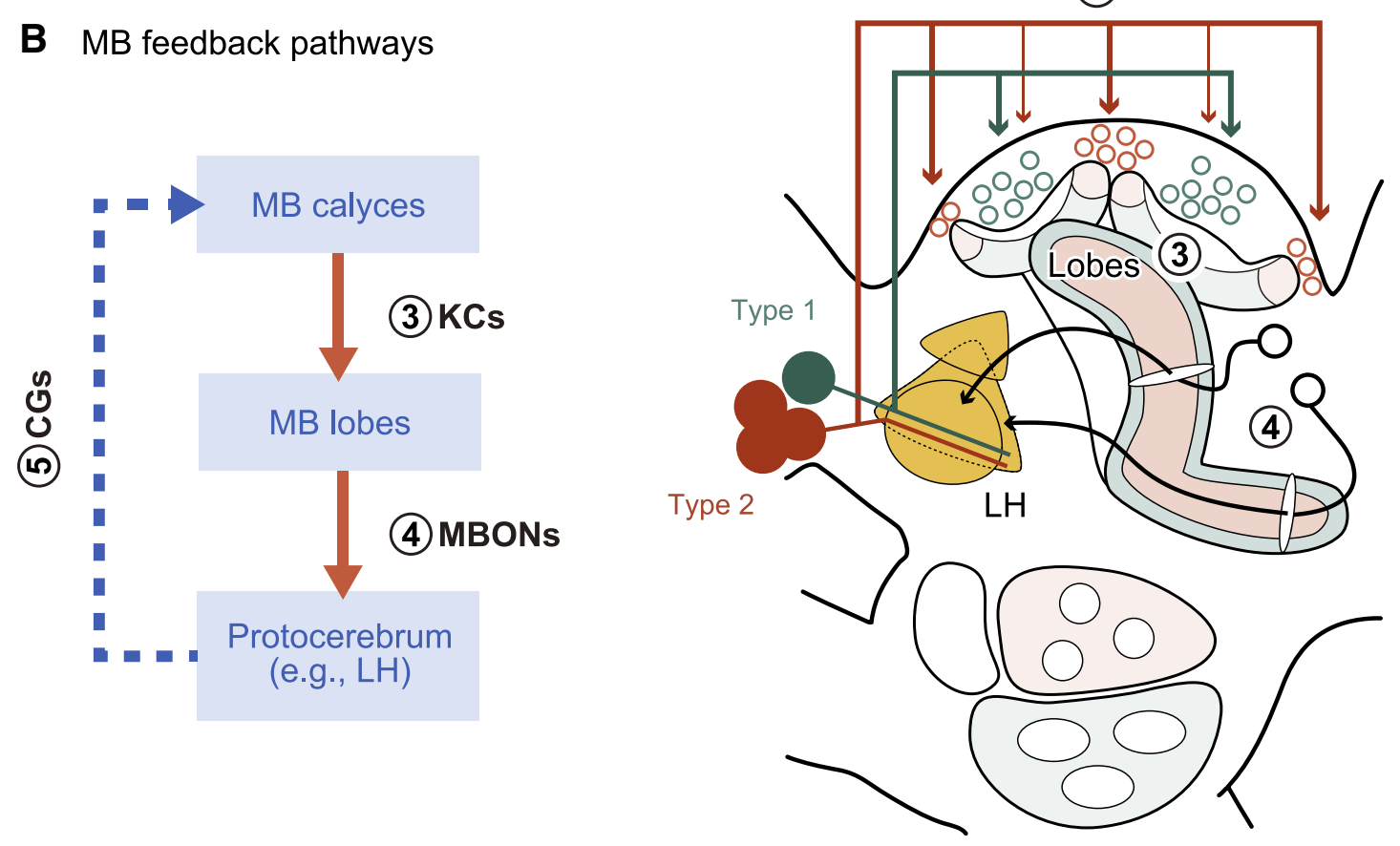

Figure 1. Schematic drawing of the parallel nature of olfactory pathways from the periphery to the $M B(\boldsymbol{A})$ and a presumed MB feedback pathway $(\boldsymbol{B})$ in the cockroach brain, evaluation of the latter being a major subject of this study. Major processing stages and related olfactory neurons are listed on the left flowcharts, in which neuron numbers (1-5) correspond to those in the right diagrams. Green and red pathways in the right diagrams represent dual processing streams reported previously: 1, 0SNs in trichoid and grooved basiconic sensilla and in perforated basiconic sensilla (Watanabe et al., 2012); 2, Type 1 and Type 2 PNs (Watanabe et al., 2017); 3, Class I and Class II KCs (Mizunami et al., 1998a,b; Strausfeld and Li, 1999b; Nishino et al., 2018); 4, MBONs and 5, Type 1 and Type 2 CGs (Takahashi et al., 2017). The lobes of the MB are omitted in the diagram (A). PED, Pedunculus; sw, single-walled-sensilla, $\mathrm{AL}$, antennal lobe; CAs, calyces; dw, double-walled sensilla.

ionization detector (model 200B, Aurora Scientific) positioned in the center of the wind tunnel, where the antenna was situated. The averaged photo-ionization detector voltage exceeded $2 \mathrm{mV}$ for $\sim 67 \mathrm{~ms}$ after the solenoid valve had been opened (total of 12 trials, 3 trials per odor), and we consider the time to be the real onset of odor stimuli.
Observation of neuron morphology

After recordings, the neurons were filled with a fluorescent dye by current injection: a constant hyperpolarizing current for LY (1-5 nA) or depolarizing current pulses $(0.25 \mathrm{~s}, 10-15 \mathrm{nA})$ at $2 \mathrm{~Hz}$ for micro-Ruby. The decapitated head was left in a humidified chamber at $4^{\circ} \mathrm{C}$ for $3-10 \mathrm{~h}$ 
Table 1. Summary of the physiological properties of 24 pairs of a CG and an MBON recorded simultaneously and used for data analysis ${ }^{a}$

\begin{tabular}{|c|c|c|c|c|c|c|c|}
\hline \multirow[b]{2}{*}{ CG/MBON } & \multirow[b]{2}{*}{ Recording site } & \multicolumn{2}{|c|}{ Firing rate $(\mathrm{Hz})$} & \multirow[b]{2}{*}{ Odor response } & \multicolumn{2}{|l|}{ Current injection } & \multirow{2}{*}{$\begin{array}{l}\text { Cross-covariance } \\
\text { (Fig. 5) }\end{array}$} \\
\hline & & Baseline & Response period & & to CG (Fig. 3) & to $M B O N$ & \\
\hline Type 1 & Dendrites & 7.2 & $48.7 \pm 10.3$ & + & - & Fig. 4 & Pair 1 \\
\hline MLONt & Dendrites & 9.7 & $54.3 \pm 14.4$ & + & & & \\
\hline Type 1 & Axon & 9.2 & $112.5 \pm 17.5$ & + & - & - & Pair 2 \\
\hline MLONt & Dendrites & 8.2 & $45.8 \pm 4.9$ & + & & & \\
\hline Type 1 & Axon & 1.2 & $18.3 \pm 1.7$ & + & $E$ (green) & - & Pair 3 \\
\hline MLONt & Dendrites & 14.8 & $63.3 \pm 4.1$ & + & & & \\
\hline Type 1 & Axon & 0.3 & $20.6 \pm 9.1$ & + & $C($ red), $D, E$ (red) Fig. 4 & - & Pair 4 \\
\hline MLONt & Dendrites & 4.5 & $56.8 \pm 7.1$ & + & & & \\
\hline Type 1 & Dendrites & 18.2 & $60.3 \pm 9.3$ & + & $C$ (blue), $D, E$ (blue) & Described in text & - \\
\hline MLONt & Dendrites & 2.2 & $30.8 \pm 9.4$ & + & & & \\
\hline Type 1 & Dendrites & 9.3 & $65.0 \pm 6.0$ & + & $E$ (light blue) & Described in text & - \\
\hline MLONt & Dendrites & 25.3 & $66.7 \pm 7.8$ & + & & & \\
\hline Type 1 & Dendrites & 22.1 & $52.0 \pm 4.3$ & + & $E$ (yellow) & - & - \\
\hline MLONt & Dendrites & 8.6 & $23.3 \pm 2.4$ & + & & & \\
\hline Type 1 & Axon & 9.7 & $56.7 \pm 7.8$ & + & $E$ (purple) & - & - \\
\hline MLONt & Dendrites & 54.5 & $58.3 \pm 6.0$ & - & & & \\
\hline Type 1 & Axon & 4.0 & $8.9 \pm 9.4$ & - & $E$ (grey) & - & - \\
\hline MLONt & Dendrites & 22.6 & $39.4 \pm 20.3$ & - & & & \\
\hline Type 1 & Dendrites & 2.9 & NA & NA & - & Described in text & - \\
\hline MLONt & Dendrites & 6.1 & NA & NA & & & \\
\hline Type 1 & Axon & 13.3 & $57.3 \pm 17.2$ & + & $G($ red) & - & Pair 5 \\
\hline JONa & Axon & 30.1 & $124.0 \pm 34.2$ & + & & & \\
\hline Type 1 & Axon & 3.5 & $39.2 \pm 5.7$ & + & $F($ red $)$ & - & - \\
\hline MLONt (left) & Dendrites & 18.8 & $17.5 \pm 8.1$ & - & & & \\
\hline Type 1 & Axon & 1.3 & $9.3 \pm 7.1$ & + & $F$ (blue) & - & - \\
\hline MLONt (left) & Dendrites & 20.6 & $17.3 \pm 9.6$ & - & & & \\
\hline NS & Axon & NA & NA & NA & $I$ (red) & - & - \\
\hline MLONt & Dendrites & 21.8 & $45.0 \pm 10.7$ & + & & & \\
\hline NS & Axon & NA & NA & NA & I (blue) & - & - \\
\hline MLONt & Dendrites & 2.1 & $45.0 \pm 5.5$ & + & & & \\
\hline NS & Dendrites & NA & NA & NA & I (green) & - & - \\
\hline MLONt & Dendrites & 19.9 & $53.3 \pm 10.2$ & + & & & \\
\hline NS & Dendrites & NA & NA & NA & I (light blue) & - & - \\
\hline MLONt & Dendrites & 16.6 & $62.9 \pm 7.9$ & + & & & \\
\hline & Dendrites & NA & NA & NA & I (yellow) & - & - \\
\hline MLONt & Dendrites & 19.3 & $79.2 \pm 8.3$ & + & & & \\
\hline NS & Axon & NA & NA & NA & I (purple) & - & - \\
\hline MLONt & Dendrites & 50.9 & $42.9 \pm 12.1$ & - & & & \\
\hline NS & Dendrites & NA & NA & NA & I (orange) & - & - \\
\hline MLONt & Dendrites & 52.3 & $53.0 \pm 20.6$ & - & & & \\
\hline NS & Axon & NA & NA & NA & $K($ red $)$ & - & - \\
\hline VLONa & Dendrites & 15.9 & $57.2 \pm 6.5$ & + & & & \\
\hline NS & Axon & NA & NA & NA & $J(\mathrm{red})$ & - & - \\
\hline MLONt (left) & Dendrites & 18.9 & $20.2 \pm 13.0$ & - & & & \\
\hline Type 2a & Dendrites & NA & NA & NA & $H($ red $)$ & - & - \\
\hline MLONt & Dendrites & 13.2 & $45.2 \pm 7.6$ & + & & & \\
\hline Type 2a & Dendrites & NA & NA & NA & $H$ (blue) & - & - \\
\hline MLONt & Dendrites & 32.4 & $54.6 \pm 7.4$ & + & & & \\
\hline
\end{tabular}

${ }^{a}$ Recording site column (axon or dendrites) represents the site of recording, judged from the magnitude of synaptic activities (Takahashi et al., 2017). Odor response column represents odor responsiveness of a spiking neuron as excitatory response $(+)$ or no response $(-)$ based on firing rate of the baseline (mean) and firing rate during the odor response period (mean \pm SD). In one pair of a Type 1 CG and an MLONt, olfactory stimulation was not performed. Odor responsiveness of an NS CG and a Type 2a CG was not analyzed (NA) (see also Determination of odor responses). Columns of current injection and cross-covariance show where the data are shown in this paper. Not all experiments could be performed in all pairs due to limited recording time. 
to let the dye diffuse throughout the neurons. The brain was dissected out, fixed in $4 \%$ formaldehyde in phosphate buffer, $\mathrm{pH} 7.4$, at $4^{\circ} \mathrm{C}$ for $6-12 \mathrm{~h}$, dehydrated in an ascending ethanol series (from $50 \%$ to $100 \%$ ), and cleared in methyl salicylate. The cleared brain was observed under a confocal laser scanning microscope (LSM 5 Pascal, Carl Zeiss) equipped with argon and helium-neon lasers. Some brains were manually crosssectioned by using a razor blade (FA-10, Feather) to allow observation under a $\times 40$ oil-immersion objective. Single neurons labeled with LY and those labeled with micro-Ruby were visualized with an argon laser $(458 \mathrm{~nm})$ and a helium-neon laser $(543 \mathrm{~nm})$, respectively. Specimens in which two neurons were differentially labeled with LY and micro-Ruby were visualized with an argon laser with a 505-530 nm bandpass filter and a helium-neon laser with a longpass filter $(>560 \mathrm{~nm})$. Images were obtained using either of two objective lenses: Plan-Apochromat $10 \times 1$ 0.75 for low-magnification images and oil-immersion Plan-Neofluar $40 \times / 1.3$ for high-magnification images. The thicknesses of optical sections were $2.3-20 \mu \mathrm{m}$ for low-magnification images and $1.0-1.2 \mu \mathrm{m}$ for high-magnification images. The resolution of optical sections was $1024 \times$ 1024 pixels. Optical sections were reconstructed two-dimensionally by image-processing software associated with the microscope (LSM Image Browser, Carl Zeiss). In addition, the highest-magnified images for $3 \mathrm{D}$ reconstruction were captured on another confocal laser scanning microscope (LSM 880 equipped with Airyscan Fast, Carl Zeiss) with a Plan-Apochromat $40 \times / 1.3$ objective lens. LY-labeled branches and micro-Ruby-labeled branches were visualized by using a $458 \mathrm{~nm}$ laser with a 495-550 $\mathrm{nm}$ bandpass filter and a $561 \mathrm{~nm}$ laser with a $570 \mathrm{~nm}$ longpass filter, respectively. The thicknesses of optical sections were 0.22 $\mu \mathrm{m}$, and the resolution was $964 \times 964$ pixels with $0.043 \mu \mathrm{m} \times 0.043 \mu \mathrm{m}$ intervals between pixels. We three-dimensionally reconstructed the highest-magnified images by using image processing software (Imaris version 9.0.0, Bitplane AG) and detected colocalization of two fluorescent signals in individual pixels (ImarisColoc module) to evaluate possible contact between differently labeled branches. Thresholds of the fluorescent signal to detect colocalization were 309.31 for LY (max intensity of 1346) by selecting $1.76 \%$ of all pixels and 3073.45 for micro-Ruby (max intensity of 13,458 ) by selecting $1.64 \%$ of all pixels. Colocalizations were displayed by white color with constant intensities. Both twodimensionally and three-dimensionally reconstructed images were processed by free photo-editing software (GNU Image Manipulation Program version 2.8.18, GIMP Development Team) to adjust input levels uniformly.

\section{Experimental design and statistical analysis}

For physiological recordings of spiking neurons, spike detection was performed on Spike2 by threshold-based feature detection script (FeatureDetect.s2s) downloaded from the Cambridge Electronic Design official website. All analysis other than spike detection was performed by using free programming software $\mathrm{R}$ version 3.6.0 ( $\mathrm{R}$ Foundation for Statistical Computing).

Determination of odor responses. Both CGs and MBONs exhibit endogenous spike activity in the absence of odor stimuli. We defined that a spiking neuron exhibited an excitatory response to odor stimuli when the following inequality was satisfied as follows:

[mean-standard deviation $(\mathrm{SD})]$ of the firing rate $(\mathrm{Hz})$

during an odor response $>$ the mean baseline rate $(\mathrm{Hz})$.

The mean firing rate $(\mathrm{Hz})$ and $\mathrm{SD}$ during an odor response were calculated from spike activities during the "odor response period" $(0.2-0.5 \mathrm{~s}$ from the real onset of odor stimulation), whereas the mean baseline rate was the averaged firing rate over continuous recording $(>5 \mathrm{~s})$ without odor stimulations (Table 1). The firing rate during the odor response period was averaged for different odors because we observed that both spiking CGs (except for a Type 2a CG) and MBONs showed similar responses to all odors used in this study, in accordance with previous observations that Type 1 and Type 2 PNs exhibit responses to the same kinds of odors, possibly due to integration of signals of different types of OSNs in the antennal lobe in cockroaches (Watanabe et al., 2017). We did not quantitatively evaluate odor responses of the Type 2a CG because they were recorded from dendrites and the spikes were not reliably separated from EPSPs.

The NS CG typically responded to odors with depolarization of membrane potential, sometimes accompanied by hyperpolarization (Takahashi et al., 2017). In this study, we did not perform quantitative evaluation of dynamics of the responses because the amplitude and time course of depolarization varied in different odors, trials, and animals, making it extremely difficult to set a quantitative criterion to evaluate the data.

Effects of current injection into a CG on spike activities of an MBON. In simultaneous recordings of a CG and an $\mathrm{MBON}$, a hyperpolarizing or depolarizing current was injected into the CG through a recording electrode $(1 \mathrm{~s}, 10-15 \mathrm{nA})$ at the same time as the start of the solenoid valve opening for odor stimulation, triggered by an electric stimulator (SEN8203 , Nihon Kohden). The triggering signal was stored in a PC. When data were acquired from a single individual, effects of current injection into a CG on spike activities of an MBON were examined by a GLM with Poisson distribution, in which a response variable was the number of spikes of an MBON during current injection into a CG. When we treated the data acquired from multiple individuals, individual-specific random effects were incorporated into the model (generalized linear mixed model [GLMM]) (Aarts et al., 2014). Fixed effects were the type of current injection into the CG [hyperpolarizing $(-)$ or depolarizing $(+)$ current injection or no injection (0)]. Statistical significance was tested by a likelihood ratio test distributed $\chi^{2}$ of the value $-2 \log (\Lambda)$, in which $\Lambda$ represents the likelihood ratio between the constructed model and the null model. The value $-2 \log (\Lambda)$ is asymptotically $\chi^{2}$ distributed with two degrees of freedom in this case (Faraway, 2006). When the effect of current injection was considered to be significant $(p<0.05)$, we further examined which type of current had a significant effect $(p<0.05)$ by the Wald test of estimated coefficients.

Postsynaptic potential induced by spikes of a presynaptic neuron. In simultaneous recordings from a dendritic branch of a Type 1 CG and an axon of an MBON with dendrites at the tip of the medial lobe (MLONt), depolarizing current was injected into the MLONt to induce a spike burst (without olfactory stimulation) and to examine whether spikes of the MLONt cause EPSPs in a dendritic branch of the CG. It was not feasible to precisely induce a single spike by a brief depolarizing current into the MLONt, probably because the branch of the MLONt is very thick and its input resistance is very low. Therefore, we injected a $0.3 \mathrm{~s}$ depolarizing current ( $3 \mathrm{nA}$ ) to cause spike bursts in the MLONt. We analyzed the data in which spikes of the MLONt accompanied no immediately preceding or following spike (from -5 to $10 \mathrm{~ms}$ relative to the peak timing of a spike), and the CG generated no spikes. In simultaneous recordings from a pair of neurons, spikes in one neuron often caused small crosstalk artifacts in the other neuron due to electrical coupling of two recording systems (Buhl et al., 2012; Watanabe et al., 2017). To reduce that kind of artifact, a differentiated spike shape of the MLONt fitted to the amplitude of the artifact was subtracted from the averaged membrane potential of the CG, following Buhl et al. (2012). Furthermore, we similarly quantified membrane potential changes of the MLONt induced by spikes of a Type 1 CG via KCs by injecting a $1 \mathrm{~s}$ depolarizing current (10 nA) into an axon of the CG coupled to odor stimulations. A crosstalk artifact in the averaged membrane potential of the MLONt was reduced in the aforementioned way.

Cross-covariance analysis of spike activities of a Type 1 CG and an $M B O N$. Cross-covariance was measured on the basis of binary spike sequences of a Type 1 CG and an MBON recorded simultaneously to quantify correlation levels between spike activities of the two neurons during spontaneous activities and during odor responses. Continuous 25-30 s recordings without odor stimuli were used as spontaneous activities and were divided into $1 \mathrm{~s}$ trials without intervals. For odor responses, a $1 \mathrm{~s}$ period from the onset of odor stimulation was defined as one trial. Odor stimuli accompanied by current injection into a CG were excluded from the analysis. We calculated cross-covariance of spike activities according to Brody (1999) and Bair et al. (2001) as follows. Each trial was divided into $1 \mathrm{~ms}$ bins representing 1 or 0 in accordance with whether it includes a spike or not. When $M$ represents the total trial number, and $t$ 
and $\tau$ represent time as the number of $1 \mathrm{~ms}$ bins, cross-covariance $C(\tau)$ is calculated as follows:

$$
C(\tau)=\frac{1}{M} \sum_{i=1}^{M} \sum_{t=1}^{1000} x_{i}(t) y_{i}(t+\tau)-\sum_{t=1}^{1000} \bar{x}(t) \bar{y}(t+\tau)
$$

where $x_{i}(t)$ and $y_{i}(t)$ represent 1 or 0 in accordance with whether the $t$ th bin of neuron $x$ and neuron $y$ in the ith trial includes a spike or not, while $\bar{x}(t)$ and $\bar{y}(t)$ are the averaged values of $x_{i}(t)$ and $y_{i}(t)$ over trials. The first term and the second term mean the raw cross-correlation and the shuffle corrector, respectively. Since $C(\tau)$ tends to increase as the firing rates of neurons increase, we divided $C(\tau)$ by the geometric mean firing rate of neuron $x$ and neuron $y$ to obtain the value $\operatorname{COV}(\tau)$, which is useful for comparing the correlation levels when firing rates are greatly different among neuron pairs (Bair et al., 2001). For convenience, we use the term "cross-covariance" for $\operatorname{COV}(\tau)$ in this paper. The correlation level between two neurons' activities was considered to be significant when $\operatorname{COV}(\tau)$ exceeded 2 times of SD at the $\tau$ th $\operatorname{bin}, \sigma(\tau)$, which is written according to Brody (1999) as follows:

$\sigma(\tau)=$

$$
\sqrt{\left(\sum_{t=1}^{1000} \sigma_{x}(t)^{2} \sigma_{y}(t+\tau)^{2}+\sum_{t=1}^{1000} \bar{x}(t)^{2} \sigma_{y}(t+\tau)^{2}+\sum_{t=1}^{1000} \sigma_{x}(t)^{2} \bar{y}(t+\tau)^{2}\right) / M}
$$

where $\sigma_{x}(t)$ and $\sigma_{y}(t)$ represent $\mathrm{SD}$ of $\bar{x}(t)$ and $\bar{y}(t)$. Other variables are the same as those in Equation 2. Cross-covariance in this paper was calculated by assigning a Type 1 CG as neuron $x$ (fixed) and an MBON as neuron $y$ (time-shifted). Auto-covariance and its SD were calculated by assigning the same neuron as both neuron $x$ and neuron $y$.

\section{Results}

Double labeling of pairs of a CG and an $\mathrm{MBON}$ revealed their close contacts in some pairs but not in other pairs

We first describe the morphologies of double-labeled pairs of a CG and an MBON to facilitate interpretation of electrophysiological data. Specifically, we focus on double labeling of the MLONt with dendrites at the tip of the medial lobe (ML) (Takahashi et al., 2017) and all four types of CGs.

An axon of the MLONt is one of the thickest among MBONs (Fig. 2A, green). Its dendritic branches are located in the entire areas of the cross-section of the ML, except for the $\gamma$ area (Fig. $2 B$ ). Since the $\gamma$ area is the termination area of Class II KCs and the rest of the cross-section area, which we refer to as the non- $\gamma$ area, is the termination area of Class I KCs (Mizunami et al., 1998a,b), the MLONt should receive synapses from Class I KCs but not from Class II KCs. It is most likely that only one MLONt resides in each brain hemisphere, as in the case of a presumable counterpart $\beta$-lobe output neuron (bLN1) in locusts (Gupta and Stopfer, 2014), since mass staining of MLONs by local dye injection into the ML $(N=4)$ revealed a cluster of three cell bodies, one of which is that of the MLONt and the other two of which are those of MLONs with distinct dendritic morphologies (MLONa, MLONb, Fig. 2C,D). Double labeling of the Type $1 \mathrm{CG}$ and the MLONt (Fig. 2A) showed extensive overlaps of branches in the $\mathrm{LH}$ as we have reported (Fig. 2I) and in the satellite neuropils anterior to the vertical lobe (VL) of the MB (Fig. 2M).

In one preparation, we performed a high-resolution analysis of confocal images. We captured images with $0.22 \mu \mathrm{m}$ optical sections in the LH and reconstructed the images three-dimensionally with software, and we extracted voxels that exhibited colocalization of fluorescent signals to visualize the sites of close contacts between differently labeled branches of the Type 1 CG and the MLONt. Occasional colocalization was detected (Fig. 2 E, F; white points indicated by black arrowheads), and it may represent synaptic connections (Pang et al., 2018).
We also 2D reconstructed branches of a pair of other types of CG (NS, Type 2a, and Type 2b CGs) and the MLONt (Fig. 2J$L, N-P)$. There were spatial overlaps for Type $2 \mathrm{a} C \mathrm{C}$ and Type $2 \mathrm{~b}$ CG (Fig. $2 K, L, O, P$ ), although the extents of overlaps were slightly less than those for Type 1 CG. Dendrites of the NS CG, on the other hand, were less overlapped with terminal branches of the MLONt (Fig. 2 J,N). Instead, dendrites of the NS CG tended to have more overlaps with terminal branches of two vertical lobe output neurons (VLONa, VLONb) in the LH (VLONa in Fig. $2 S, T$; VLONb data not shown). Dendrites of a Type 1 CG had much less spatial overlaps with terminal branches of another VLON (VLONt with dendrites at the tip of VL) than with those of the MLONt, as we have reported (Figure 2Q, $R$, modified from Takahashi et al., 2017). We observed dendritic branches of these VLONs in the non- $\gamma$ area but not in in the $\gamma$ area, suggesting that they receive synapses from Class I KCs. In summary, all of the stained MBONs with terminals in the $\mathrm{LH}(N=21)$ had dendrites in the non- $\gamma$ area and hence were most likely to receive synapses from Class I KCs. We found no MBONs with dendrites in the $\gamma$ area; thus, it remains unknown to which CGs these neurons are connected.

\section{A CG strongly inhibits odor responses of some MBONs but not those of other MBONs}

We investigated whether CGs could indeed inhibit spike activities of MBONs, which receive synapses from $\mathrm{KCs}$, to achieve gain control in the $\mathrm{MB}$ by simultaneous recordings of a CG and an MBON. CGs exhibited excitatory responses to almost all kinds of odor (Takahashi et al., 2017). Here we injected a $1 \mathrm{~s}$ current into a CG at the same time as a 0.5 s olfactory stimulation and investigated the effect on spike activities of an MBON (Fig. $3 A, B$ ). Figure $3 C, D$ shows the results for two pairs of a Type $1 C G$ and an MLONt, in which spikes of both neurons could be counted during current injection (Fig. 3-1, available at https://doi.org/ 10.1523/JNEUROSCI.0088-19.2019.f3-1). The number of spikes of the MLONt during current injection into the Type 1 CG was fitted well against the number of spikes of the Type 1 CG by GLMM of Poisson regression with individual-specific random effects (Fig. $3 C ; \chi^{2}=158, p=4.0 \mathrm{e}-36$ when the constructed GLMM was compared with the null model by likelihood ratio test distributed $\chi^{2}$ ). Inhibition or activation of spike activities of the Type 1 CG during odor responses resulted in an increase or decrease in spike activities of the MLONt for 300-500 ms or 320-730 ms (Fig. $3 D$ ), respectively, demonstrating that activities of the Type $1 \mathrm{CG}$ indeed control the magnitude of odor responses of the MLONt.

For quantitative investigation of the effects of current injection into the Type 1 CG on activities of MLONt, we evaluated the data from seven pairs by GLMM (Fig. 3E; Table 2; for the detailed information of stimulus and the neural response, see also Fig. 3-1, available at https://doi.org/10.1523/JNEUROSCI.0088-19.2019. f3-1). We first statistically confirmed that current injections into a Type 1 CG, either hyperpolarizing or depolarizing, significantly affected spike activities of an MLONt (Fig. $3 E ; N=7, \chi^{2}=287$, $p=4.0 \mathrm{e}-63$, likelihood ratio test distributed $\chi^{2}$ ), and we then separately tested the effects of depolarizing and hyperpolarizing current injections. We found that both hyperpolarizing $(-)$ and depolarizing $(+)$ current injections into a Type 1 CG significantly increased or decreased spike activities of an MLONt, respectively (Table $2 ;(-): z=4.487, p=7.2 \mathrm{e}-06$, Wald test; $(+): z=-12.92$, $p=3.5 \mathrm{e}-38$, Wald test). Among seven pairs of a Type $1 \mathrm{CG}$ and an MLONt, one Type 1 CG (gray symbol) and two MLONts (opened symbol) did not exhibit excitatory odor responses. Exclusion of these data from analysis did not alter the statistical results. Cur- 

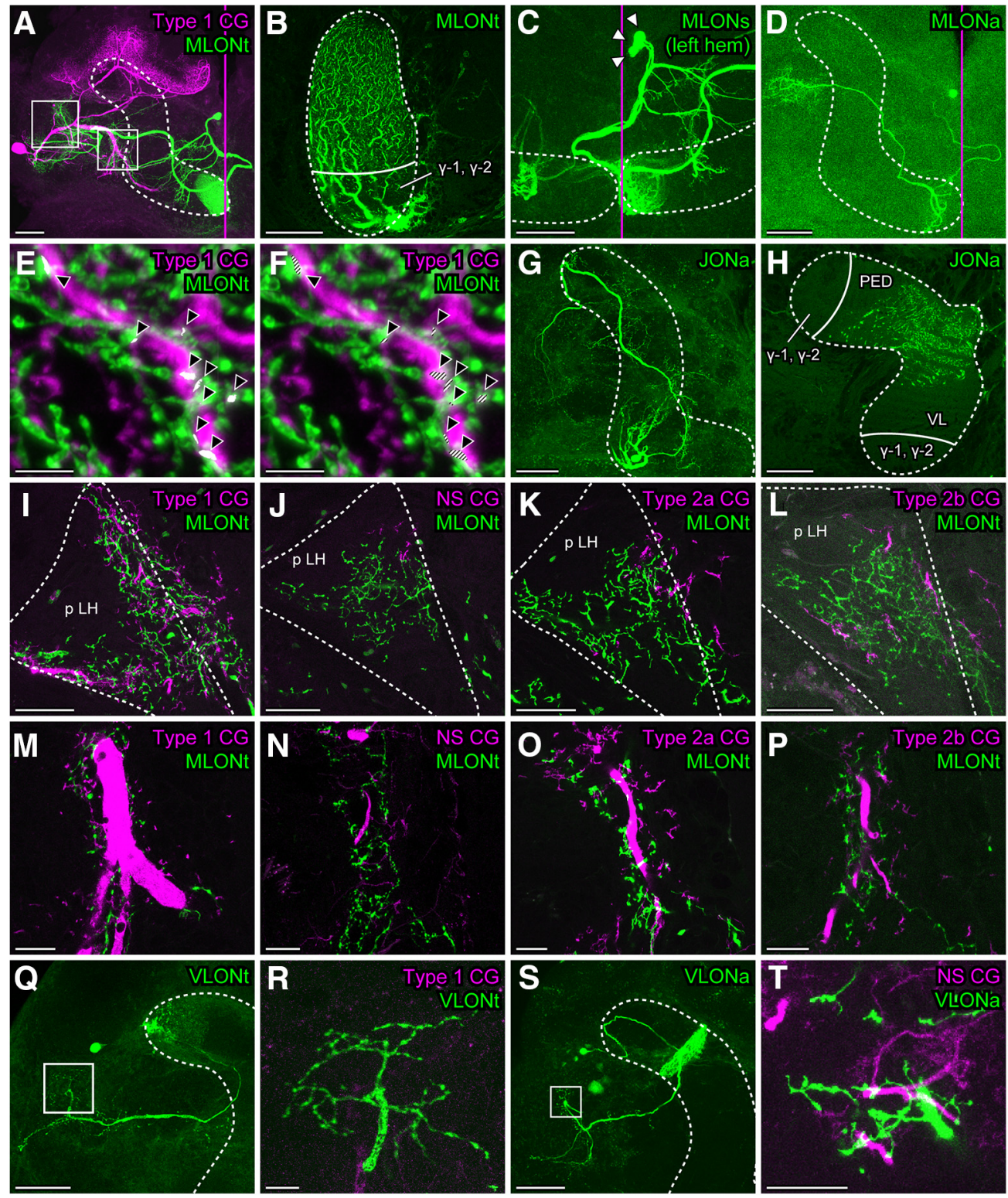

Figure 2. Close appositions of dendrites of (Gs and terminal branches of MBONs. A, A low-magnification confocal image of neurons labeled with different dyes: a Type 1 CG (magenta) and an MLONt (green) with dendrites located in the tip of the ML. White rectangles represent the posterior LH (p LH) (left) and the satellite neuropils anterior to the VL (right), which are magnified in the following panels. $\boldsymbol{B}$, Dendritic branches of an MLONt in a sagittal section of the ML viewed medially. $\boldsymbol{C}, \boldsymbol{D}$, The MLONt could be discriminated from other MLONs. Dye injection into the ML of the left hemisphere revealed a cluster of three cell bodies ( $\boldsymbol{C}$, white arrowheads). One is MLONt. Another is MLONa that has dendrites just lateral to the tip of the ML, the overall morphology of which was revealed by intracellular staining in another individual (D). The third is MLONb for which dendrites are located also in the contralateral MB. E, $\boldsymbol{F}$, Frontal 3D views of branches of the Type 1 CG (magenta) and the MLONt (green) in the $\mathrm{p} \mathrm{LH} \mathrm{reconstructed} \mathrm{from} \mathrm{high-resolution} \mathrm{optical} \mathrm{sections.} \mathrm{White} \mathrm{points} \mathrm{indicated} \mathrm{by} \mathrm{black} \mathrm{arrowheads} \mathrm{show} \mathrm{colocalizations} \mathrm{of} \mathrm{branches} \mathrm{of} \mathrm{the} \mathrm{two} \mathrm{neurons,}$ detected by image processing software. $F$, White points of colocalization are shaded to discriminate them from white-like points where magenta and green voxels are overlaid into one pixel in a $2 D$ view. $\boldsymbol{G}, \boldsymbol{H}$, Morphologies of a JONa, a type of MBON with dendrites located in the junction between the VL and the ML. $\boldsymbol{H}$, Cross section of the pedunculus (PED) and VL. I-P, Differently labeled branches of a CG (magenta) and an MLONt (green) in the p LH $(\boldsymbol{I}-\boldsymbol{L})$ and the satellite neuropils anterior to the VL $(\boldsymbol{M}-\boldsymbol{P})$. $\boldsymbol{Q}, \boldsymbol{R}$, Differently labeled branches of the Type $1 \mathrm{CG}$ (magenta) and a VLONt (green), the overall morphology of which is shown in Q.S, $\boldsymbol{I}$, Differently labeled branches of an NS CG (magenta) and VLONa (green) (T), the overall morphology of which is shown in $S$. White dashed lines in all panels indicate the lobes of the $M B$, except in $I-L$ where they indicate the $\mathrm{p} L$. Magenta vertical lines indicate the midline of the brain. The numbers and depths of confocal sections used for 2D or 3D reconstructions are as follows: (A) 49 sections at $364 \mu \mathrm{m},(\boldsymbol{B}) 115$ sections at $114 \mu \mathrm{m},(\boldsymbol{C}) 90$ sections at $267 \mu \mathrm{m},(\boldsymbol{D}) 38$ sections at $185 \mu \mathrm{m},(\boldsymbol{E}, \boldsymbol{F}) 83$ sections at $18.1 \mu \mathrm{m},(\boldsymbol{G}) 143$ sections at $326.6 \mu \mathrm{m},(\boldsymbol{H}) 3$ sections at $6.97 \mu \mathrm{m},(\boldsymbol{I}) 4$ sections at $3.3 \mu \mathrm{m},(\boldsymbol{J}) 6$ sections at $5.5 \mu \mathrm{m},(\boldsymbol{K}) 4$ sections at $3.3 \mu \mathrm{m},(\boldsymbol{L}) 6$ sections at $5.5 \mu \mathrm{m},(\boldsymbol{M}) 4$ sections at $3.3 \mu \mathrm{m},(\boldsymbol{N}) 7$ sections at $6.6 \mu \mathrm{m},(\mathbf{O}) 4$ sections at $3.3 \mu \mathrm{m},(\boldsymbol{P}) 4$ sections at $3.3 \mu \mathrm{m},(\mathbf{Q}) 50$ sections at $294 \mu \mathrm{m},(\boldsymbol{R}) 10$ sections at $54 \mu \mathrm{m},(\boldsymbol{S}) 20$ sections at $380 \mu \mathrm{m}$, and $(\boldsymbol{T}) 9$ sections at $9.6 \mu \mathrm{m}$. Scale bars: $\boldsymbol{A}, \boldsymbol{C}, \boldsymbol{D}, \mathbf{G}, \mathbf{Q}$, $\mathbf{S}, 100 \mu \mathrm{m} ; \boldsymbol{B}, \boldsymbol{H}-\boldsymbol{L}, \boldsymbol{R}, 50 \mu \mathrm{m} ; \boldsymbol{M}-\boldsymbol{P}, \boldsymbol{T}, 20 \mu \mathrm{m} ; \boldsymbol{E}, \boldsymbol{F}, 5 \mu \mathrm{m}$. $\mathbf{Q}, \boldsymbol{R}$, Modified with permission from Takahashi et al. (2017).

rent injection into a Type 1 CG did not affect spike activities of an MLONt in the contralateral (left) hemisphere (Fig. $3 F ; N=2$, $\left.\chi^{2}=0.188, p=0.91\right)$. This was in accordance with the fact that the contralateral (left) MLONt exhibited no responses to odor stimuli applied to the right antenna and its neurites are confined to the left hemisphere.
We also found that current injection into the Type 1 CG significantly affected spike activities of a junction output neuron (JONa), another type of MBON (Fig. $3 G ; N=1, \chi^{2}=137, p=$ 2.2e-30). Dendrites of JONa are located in a part of the non- $\gamma$ area in the cross-section of the junction between the VL and the ML (Fig. 2H), suggesting that it receives synaptic input from 
A

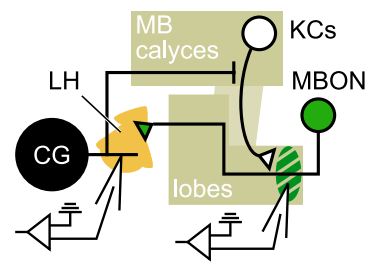

B
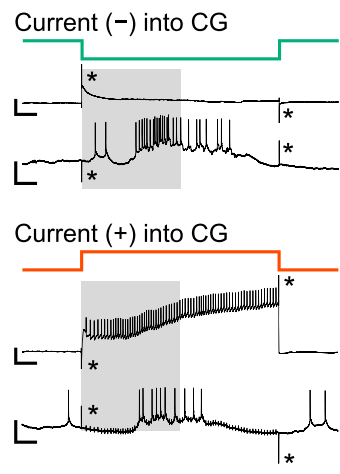

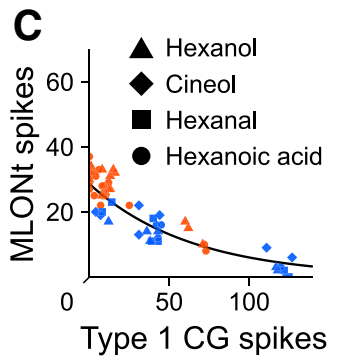

E Type 1 CG + MLONt
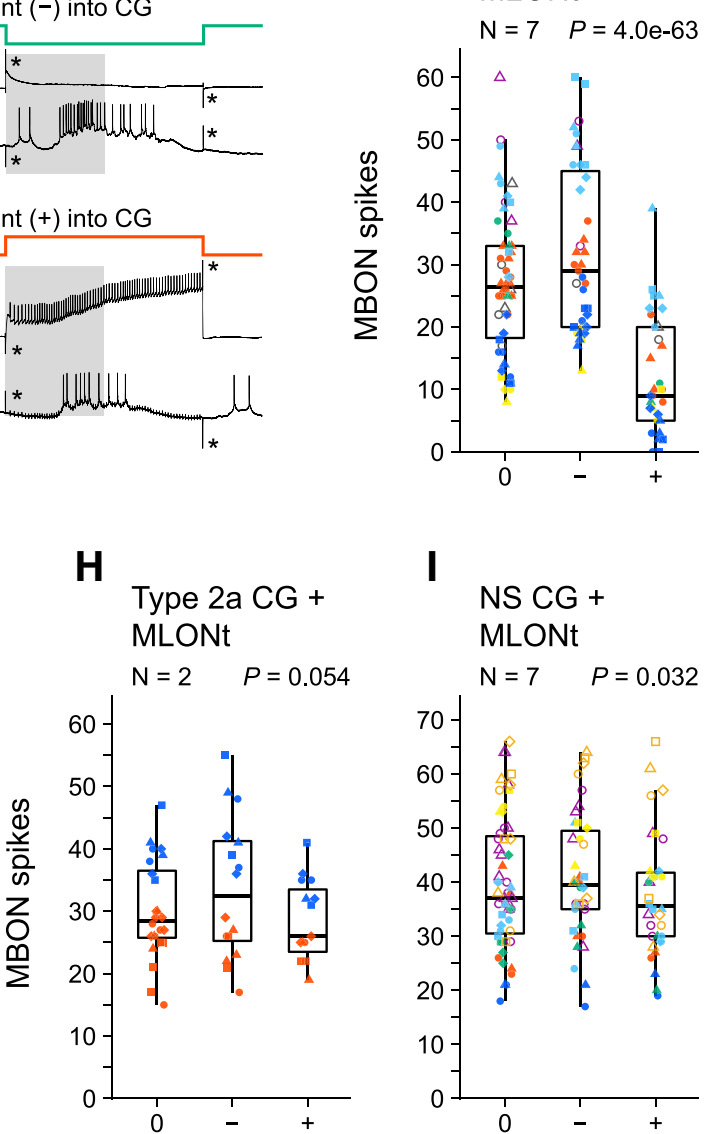

\section{I}

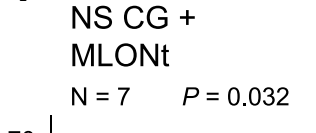

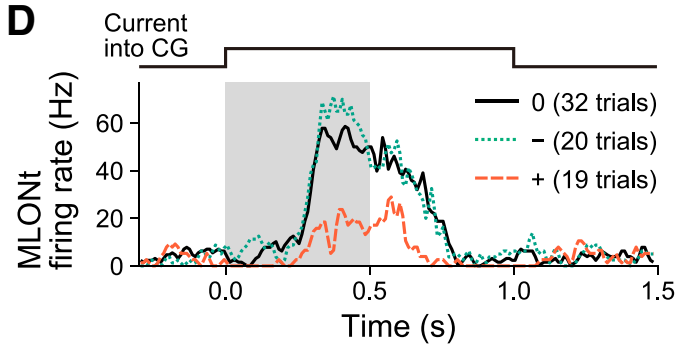

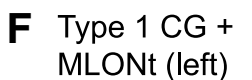

G Type 1 CG + $\mathrm{JONa}$
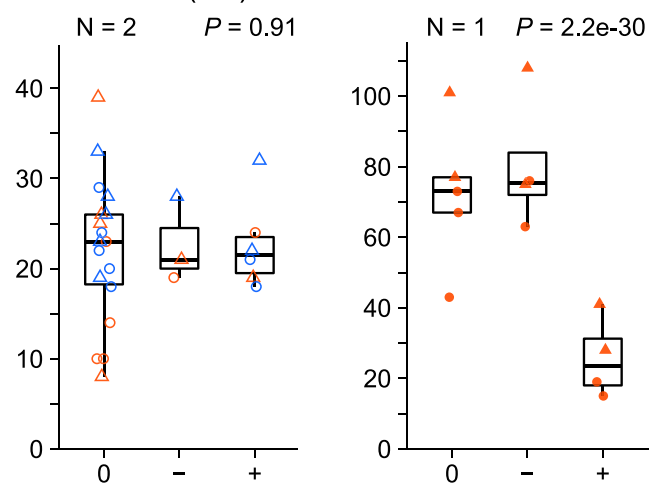

Current injection into CG

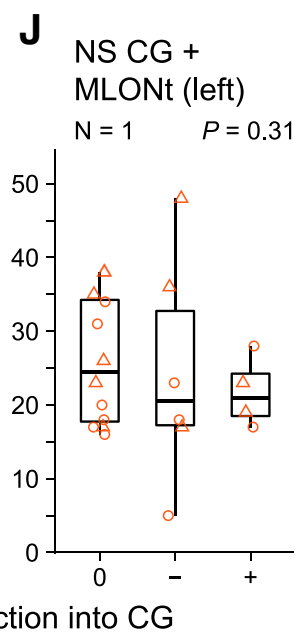

K VLONa

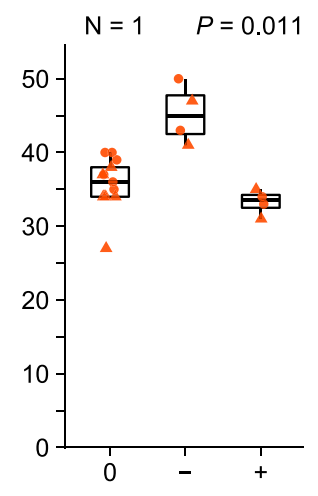

Figure 3. Effects of hyperpolarizing or depolarizing current injection into a $C G$ on spike activities of an MBON. A, Schematic diagram of the experiment. Recording electrodes were inserted in a dendritic branch or an axonal branch of a CG and an MBON. B, Sample recordings of a 1 s hyperpolarizing (top) or depolarizing (bottom) current injection into a Type 1 (G. A current injection was triggered at the same time as opening of the solenoid valve for 0.5 s odor stimulation (gray rectangles). In each recording, the top trace is the Type 1 CG and the bottom trace is the MLONt. Calibration: vertical, $50 \mathrm{mV}$ in CG traces, $10 \mathrm{mV}$ in MLONt traces; horizontal, $0.1 \mathrm{~s}$. *Artifacts induced by current injection. C, Numbers of spikes of an MLONt during a $1 \mathrm{~s} \mathrm{current} \mathrm{injection} \mathrm{into} \mathrm{a} \mathrm{Type} 1$ CG plotted against those of the Type 1 CG. One symbol represents one trial, and differently colored symbols represent recordings from different individuals. Trials in which the membrane potential of the CG was saturated were excluded. The best-fitted curve estimated by Poisson regression with individual-specific random effects is shown ( $N=2$, no current: $32,-: 13,+: 14, p=4.0 \mathrm{e}-36$, likelihood ratio test distributed $\chi^{2}$ ). D. Time course of spike activities of the MLONts shown in $\boldsymbol{C}$. Firing rate was calculated from $10 \mathrm{~ms}$ binned data and smoothed by a Gaussian filter ( $\left.\sigma=0.8\right)$. Gray rectangle represents $0.5 \mathrm{~s}$ solenoid valve opening for odor stimulation. $\boldsymbol{E}-\boldsymbol{K}$, Spike frequencies of MBONs during a $1 \mathrm{~s}$ current injection into the Type 1 CG $(\boldsymbol{E}-\boldsymbol{G})$, Type 2 a CG $(\boldsymbol{H})$, or the NS CG $(\boldsymbol{I}-\boldsymbol{K})$. Differently colored symbols in each panel represent recordings from different individuals. $\boldsymbol{E}$, Red and blue symbols represent data of the pairs shown in $\boldsymbol{C}, \boldsymbol{D}$. A neuron with no excitatory responses to odors is represented by gray (CG) or open (MBON) symbols. $p$ values of likelihood ratio test distributed $\chi^{2}$ are shown at the top of each graph. Numbers of trials in each experiment are as follows: (E) no current: $58,-: 39,+: 33 ;(\boldsymbol{F})$ no current: $18,-: 3,+: 6 ;(\boldsymbol{G})$ no current: $5,-: 4,+: 4 ;(\boldsymbol{H})$ no current: $24,-: 16,+: 15 ;(\boldsymbol{I})$ no current: 59, $-: 38,+: 34 ;(\boldsymbol{I})$ no current: 12, $-: 6,+: 4 ;(\boldsymbol{J})$ no current: $13,-: 4,+: 4$. For a summary of the statistical results of the experiments, see Table 2 . For detailed information on the stimulus and neural response in each trial, see Figure 3-1 (available at https://doi.org/10.1523/JNEUROSCI.0088-19.2019.f3-1).

Class I KCs. Terminal arborizations of JONa are located in the LH and the satellite neuropils anterior to the lobes (Fig. $2 G$ ), which appeared to have some overlap with dendrites of a Type 1 CG. Further statistical evaluation showed that depolarizing current injection significantly decreased spike activities of JONa (Table 2; $z=-9.229, p=2.7 \mathrm{e}-20)$, but the significant effect of hyperpolarizing current injection was not detected (Table 2; $z=1.420$, $p=0.16)$.

Next, we performed current injections into an NS CG. An NS CG does not generate spikes and responds to odor stimulation 
Table 2. Statistical results for the effects of current injection into a CG on spike activities of an MBON (Fig. 3) ${ }^{a}$

\begin{tabular}{|c|c|c|c|c|c|c|c|c|}
\hline$\overline{C G}$ & MBON & Pairs $(M)$ & Likelihood ratio test & $-1+$ & Coefficient & SE & $z$ & Wald test \\
\hline \multirow[t]{7}{*}{ Type 1} & \multirow[t]{2}{*}{ MLONt } & \multirow[t]{2}{*}{7} & \multirow[t]{2}{*}{$\chi^{2}=287, p=4.0 \mathrm{e}-63$} & - & 0.175 & 0.039 & 4.487 & $p=7.2 \mathrm{e}-06$ \\
\hline & & & & + & -0.736 & 0.057 & -12.92 & $p=3.5 \mathrm{e}-38$ \\
\hline & \multirow[t]{2}{*}{ (pairs with odor responses) } & \multirow[t]{2}{*}{5} & \multirow[t]{2}{*}{$\chi^{2}=298, p=2.4 \mathrm{e}-65$} & - & 0.224 & 0.043 & 5.211 & $p=1.9 \mathrm{e}-07$ \\
\hline & & & & + & -0.753 & 0.060 & -12.46 & $p=1.2 \mathrm{e}-35$ \\
\hline & \multirow[t]{2}{*}{ JONa } & \multirow[t]{2}{*}{1} & \multirow{2}{*}{$\chi^{2}=137, p=2.2 \mathrm{e}-30$} & - & 0.109 & 0.077 & 1.420 & $p=0.16$ \\
\hline & & & & + & -1.031 & 0.112 & -9.229 & $p=2.7 \mathrm{e}-20$ \\
\hline & MLONt (left) & 2 & $\chi^{2}=0.188, p=0.91$ & NA & & & & \\
\hline \multirow[t]{6}{*}{ NS } & \multirow[t]{2}{*}{ MLONt } & \multirow[t]{2}{*}{7} & \multirow[t]{2}{*}{$\chi^{2}=6.87, p=0.032$} & - & 0.025 & 0.033 & 0.756 & $p=0.45$ \\
\hline & & & & + & -0.071 & 0.035 & -1.997 & $p=0.046$ \\
\hline & (pairs with odor responses) & 5 & $\chi^{2}=3.05, p=0.22$ & NA & & & & \\
\hline & \multirow[t]{2}{*}{ VLONa } & \multirow[t]{2}{*}{1} & \multirow[t]{2}{*}{$\chi^{2}=8.97, p=0.011$} & - & 0.235 & 0.088 & 2.684 & $p=7.3 \mathrm{e}-03$ \\
\hline & & & & + & -0.073 & 0.098 & -0.743 & $p=0.46$ \\
\hline & MLONt (left) & 1 & $\chi^{2}=2.37, p=0.31$ & NA & & & & \\
\hline Type 2a & MLONt & 2 & $\chi^{2}=5.82, p=0.054$ & NA & & & & \\
\hline
\end{tabular}

${ }^{a}$ When the effect of current injection $(10-15 \mathrm{nA})$ into a $\left(\mathrm{G}\right.$ was significant $\left(~ p<0.05\right.$ by likelihood ratio test $\left.\chi^{2}\right)$, the significance of a hyperpolarizing $(-)$ or depolarizing $(+)$ current was analyzed by the Wald test. Coefficient, Estimated coefficient of a GLM or a GLMM; SE, standard error of coefficient; $z$, statistic value for the Wald test calculated from the coefficient value and its SE.

with graded membrane potential changes (Takahashi et al., 2017). Its typical odor response is a transient depolarization sometimes followed by a hyperpolarization, with the amplitude and duration of depolarization varying among individuals and even during recording of the same neuron. Current injection into an NS CG induced a weak but statistically significant effect on spike activities of the MLONt in the ipsilateral hemisphere (Fig. 3I; $N=7, \chi^{2}=6.87, p=0.032$ ); depolarizing current injection significantly deceased spike activities of the MLONt but much more moderately than that to a Type 1 CG (Table 2; $z=-1.997$, $p=0.046$ ), whereas hyperpolarizing current injection had no significant effect (Table $2 ; z=0.756, p=0.45$ ). As expected, no significant effect was detected in a pair of the NS CG and the MLONt in the contralateral hemisphere (Fig. 3J; $N=1, \chi^{2}=$ 2.37, $p=0.31$ ). Current injection into an NS CG significantly affected spike activities of a VLONa (Fig. $3 K ; N=1, \chi^{2}=8.97$, $p=0.011$ ). Hyperpolarizing current injection into the NS CG significantly increased spike activities of VLONa (Table $2 ; z=2.684$, $p=7.3 \mathrm{e}-3$ ), whereas a significant effect of depolarizing current injection was not detected (Table $2 ; z=-0.743, p=0.46$ ).

We also performed current injections into a Type 2a CG. The Type 2a CG generated a smaller number of spikes than did the Type 1 CG during spontaneous activity and during odor response (Takahashi et al., 2017). The effect of current injection into a Type 2a CG on spike activities of an MLONt in the ipsilateral hemisphere was marginal, with the $p$ value being just above the significance level (Fig. $3 H ; N=2, \chi^{2}=5.82, p=$ $0.054)$. Thus, although Type 1 CG strongly inhibits MLONt, the effect of an NS CG and Type 2a CG on MLONt was weak or absent.

\section{Evidence of excitatory connections from MLONt to Type 1 CG}

To determine whether individual spikes of the MLONt induce synaptic potentials in a Type $1 \mathrm{CG}$, we induced a burst of spike activities in the MLONt by injecting a depolarizing current $(N=$ 4; for a representative example, see Fig. 4A). We aligned the membrane potential of the Type 1 CG to the peak of each spike in spike bursts of the MLONt, but no clear membrane potential changes were observable in single traces (Fig. $4 B$ ). Moreover, a small crosstalk artifact (asterisks) interfered with observations of EPSPs. When we averaged 250 traces, however, a small depolarization was observed following a crosstalk artifact (Fig. 4C). The depolarization was more remarkable when the artifact was par- tially eliminated by subtracting the differentiated action potential of the MLONt (Fig. 4C, red line) with the amplitude fitted to that of the artifact. At $2.0 \mathrm{~ms}$ after the peak of the spike of the MLONt, the depolarization reached $0.226 \mathrm{mV}$ in amplitude, with peak depolarization of $0.239 \mathrm{mV}$ at $3.6 \mathrm{~ms}$ (Fig. 4D). Similar depolarization was detected in a Type 1 CG in three other pairs with an MLONt, with the peak amplitude differing among pairs $(0.156$, 0.064 , and $0.045 \mathrm{mV}$ ). These observations provide physiological evidence of direct synaptic input from the MLONt to the Type 1 CG. The smallness of the recorded EPSPs is best accounted for by that the Type 1 CG receives synapses from a large number of MBONs, with the MLONt being only one of these neurons.

We reanalyzed the paired recordings shown in Figure $3 C$ to examine whether each spike of a Type 1 CG produces a hyperpolarizing potential in an MLONt (Fig. 4E). We used one of two pairs (Fig. 3C, red individual) for this analysis since the spikes were fewer in another pair. When the membrane potential of the MLONt was aligned to the peak of each spike of the Type 1 CG, there was a small hyperpolarization after a crosstalk artifact in single traces (Fig. 4F). The hyperpolarization was more remarkable after averaging 284 traces (Fig. $4 G$, black line). In this recording, subtracting a differentiated spike shape of the Type 1 CG (Fig. 4G, red line) was less effective for eliminating the crosstalk artifact, but a hyperpolarization was clearly visible following the artifact, with the trough $-0.253 \mathrm{mV}$ at $6.7 \mathrm{~ms}$ after the peak of the spike of the Type 1 CG. It is most likely that this hyperpolarization is produced by that a spike of the Type 1 CG inhibited spike responses of a population of KCs to odors and that this led to a reduction of excitatory synaptic transmission from KCs to the MLONt.

\section{Cross-covariance analysis of spike activities of pairs of a CG and an MBON}

We then performed cross-covariance analysis of spike activities of pairs of a Type 1 CG and an MLONt. A Type 1 CG typically exhibited endogenous spike activities comprising episodic bursts of spikes, even without odor stimulation (Takahashi et al., 2017), and the MLONt also exhibited endogenous spike activities. We frequently observed coincident spike burst activities between a Type 1 CG and an MLONt when recorded simultaneously (Fig. 5, insets), although some spike bursts and solitary spikes seen in the MLONt were occasionally absent in the Type 1 CG. We measured cross-covariance of binary spike sequences of the pair of neurons during spontaneous activities and during their responses to odor stimulations. Cross-covariance represents shuffle-corrected 
A
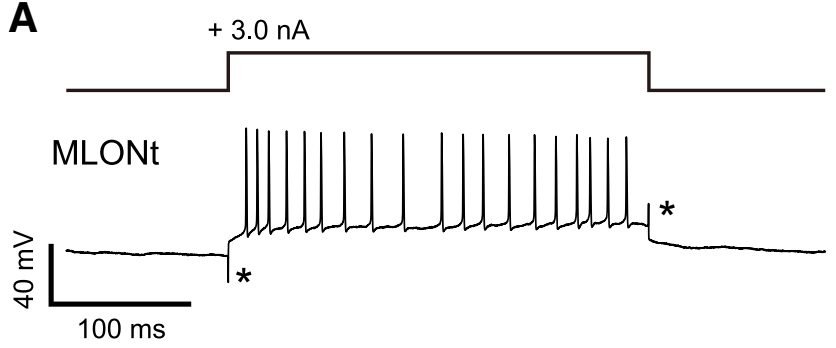

B

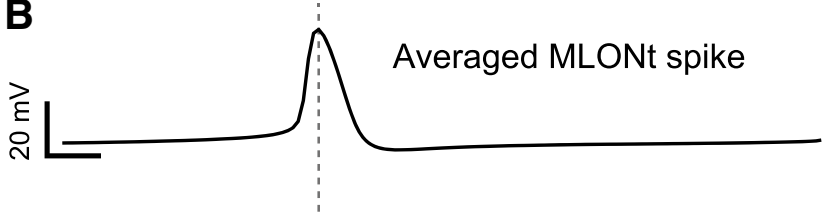

Type 1 CG membrane potential traces

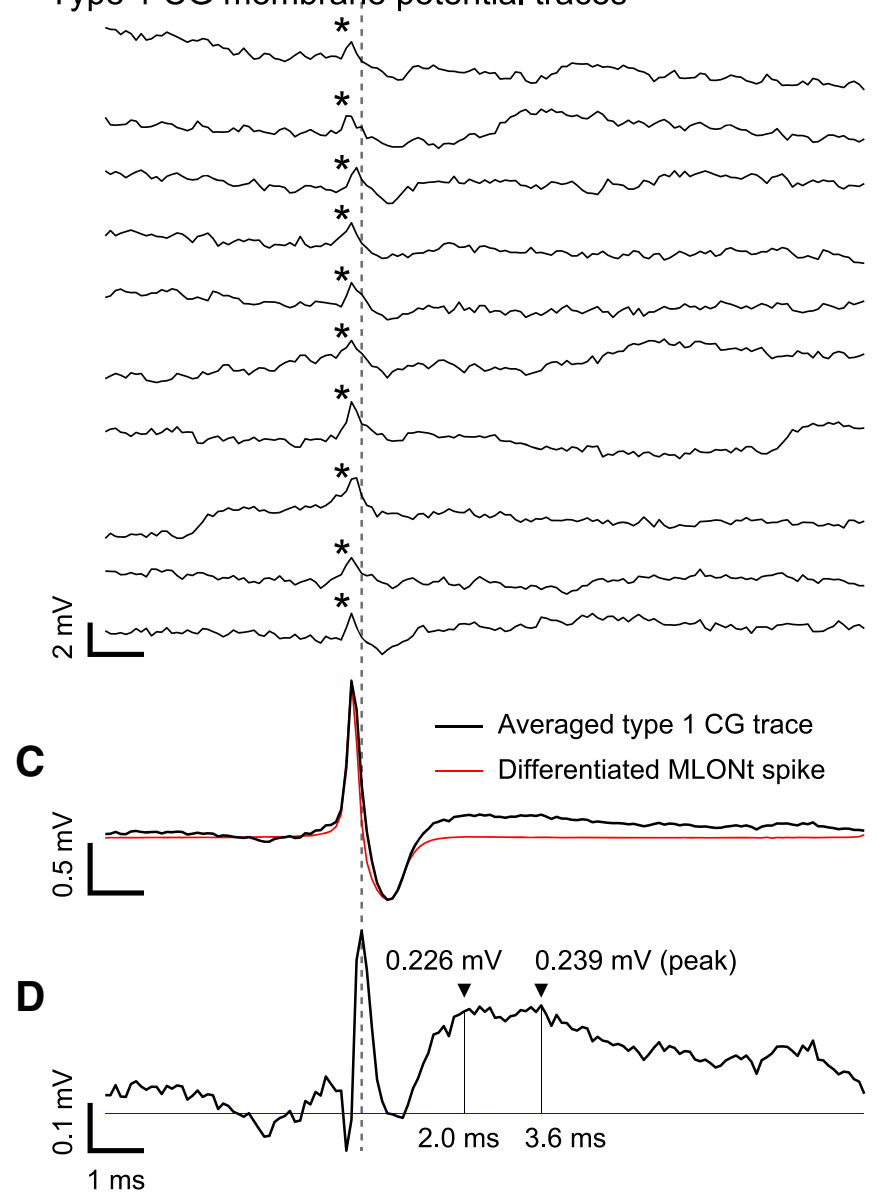

E
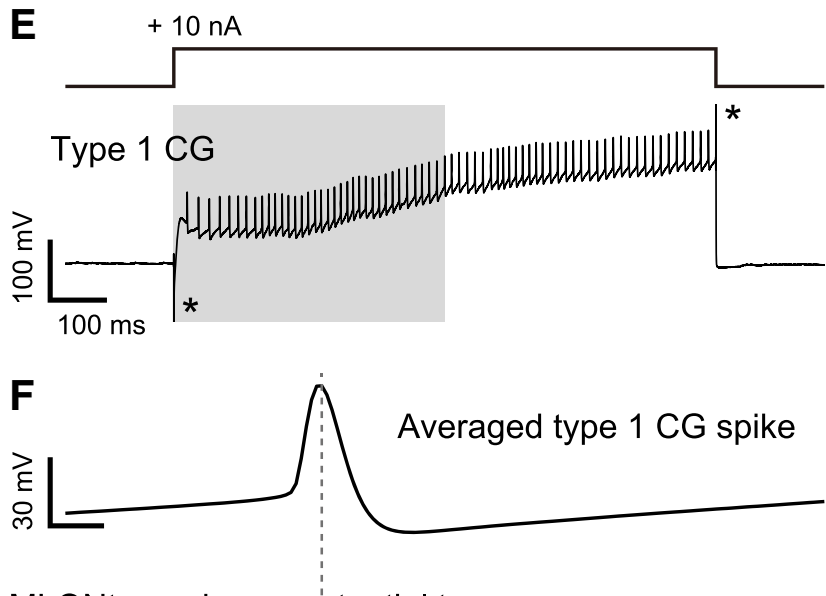

MLONt membrane potential traces

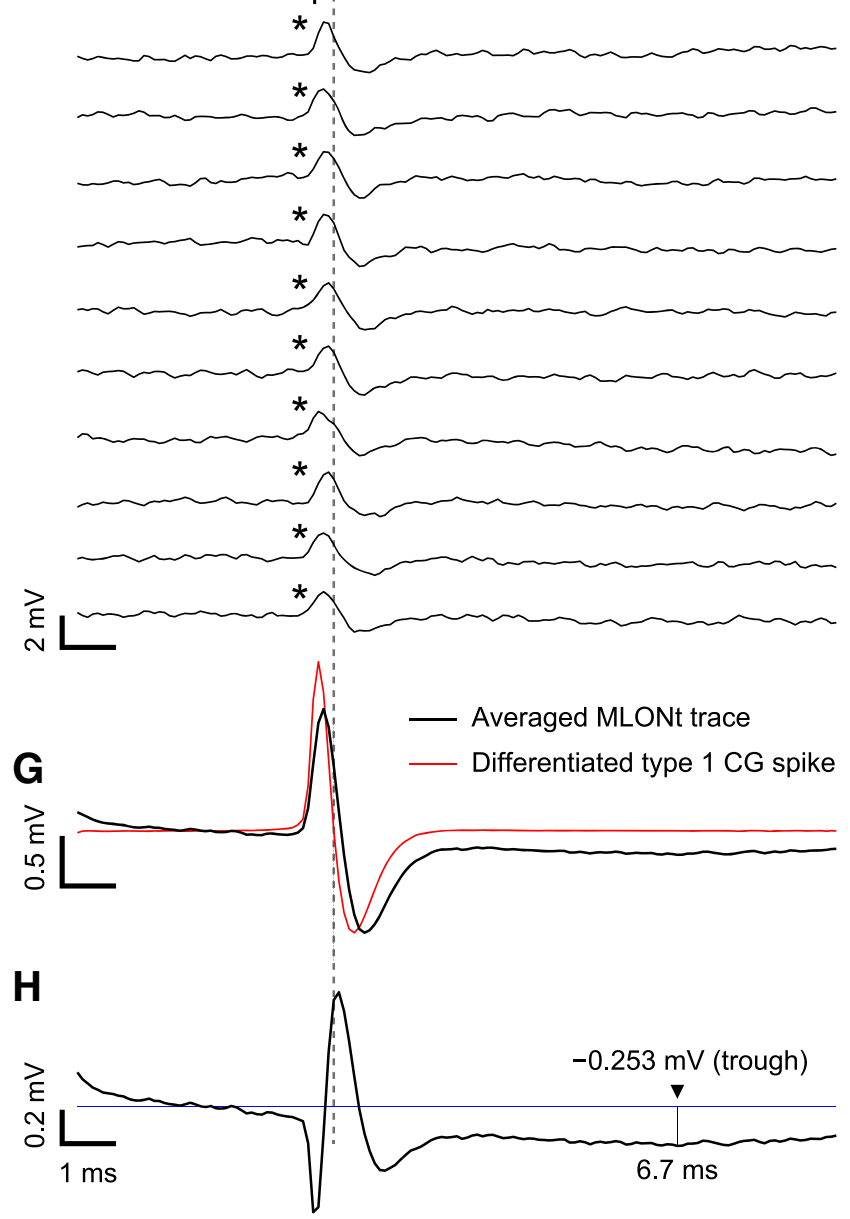

Figure 4. An EPSP of a Type 1 CG produced by a spike of an MLONt $(\boldsymbol{A}-\boldsymbol{D})$ and a hyperpolarizing potential of the MLONt produced by a spike of the Type $1(\mathrm{CG}(\boldsymbol{E}-\boldsymbol{H})$. $\boldsymbol{A}, \boldsymbol{E}, \mathrm{Spike}$ burst activities were induced by depolarizing current injection into the MLONt $(\boldsymbol{A})$ or the Type $1(G(E)$. A current injection into the $(G$ was triggered at the same time as opening of the solenoid valve for 0.5 s odor stimulation ( $\boldsymbol{E}$, gray rectangle). ${ }^{*}$ Artifacts caused by current injection. $\boldsymbol{B}, \boldsymbol{F}$, Averaged potentials of spikes induced by depolarizing current injection are shown at the top (averaged $>250$ spikes in $\boldsymbol{B}$ and 284 spikes in $\boldsymbol{F}$ ). Typical traces of membrane potential of a downstream neuron (Type $1(\mathrm{G}$ in $\boldsymbol{B}$ or MLONt in $\boldsymbol{F}$ ) are aligned to the peak of the averaged spike (vertical dashed line). ${ }^{*}$ Artifacts caused by crosstalk between the recording electrodes. $C, G$, Averaged trace of the membrane potential of the downstream neuron (black) and the differentiated potential of the averaged spike of the MLONt $(\boldsymbol{C})$ or the Type $1(G(G)$ (red), the amplitude of which was fitted to that of the artifact based on the ratio of negative peak amplitudes. $\boldsymbol{D}, \boldsymbol{H}$, Differentiated potential of the averaged spike was subtracted from averaged membrane potential of the downstream neuron for reduction of the artifact. Although the artifact was not completely eliminated, reduction of the artifact allowed detection of a small membrane potential change following a spike of the MLONt (D) or the Type $1(\mathbf{C G}(\boldsymbol{H})$. Horizontal blue line indicates the baseline of the membrane potential. In the Type 1 CG (D), depolarization was $0.226 \mathrm{mV}$ in amplitude at $2.0 \mathrm{~ms}$ after the peak of the spike of the MLONt, which is almost the same as the peak depolarization of $0.239 \mathrm{mV}$ at $3.6 \mathrm{~ms}$. In the MLONt $(\boldsymbol{H})$, hyperpolarization was $-0.253 \mathrm{mV}$ in amplitude at $6.7 \mathrm{~ms}$ after the peak of the spike of the Type 1 CG. 

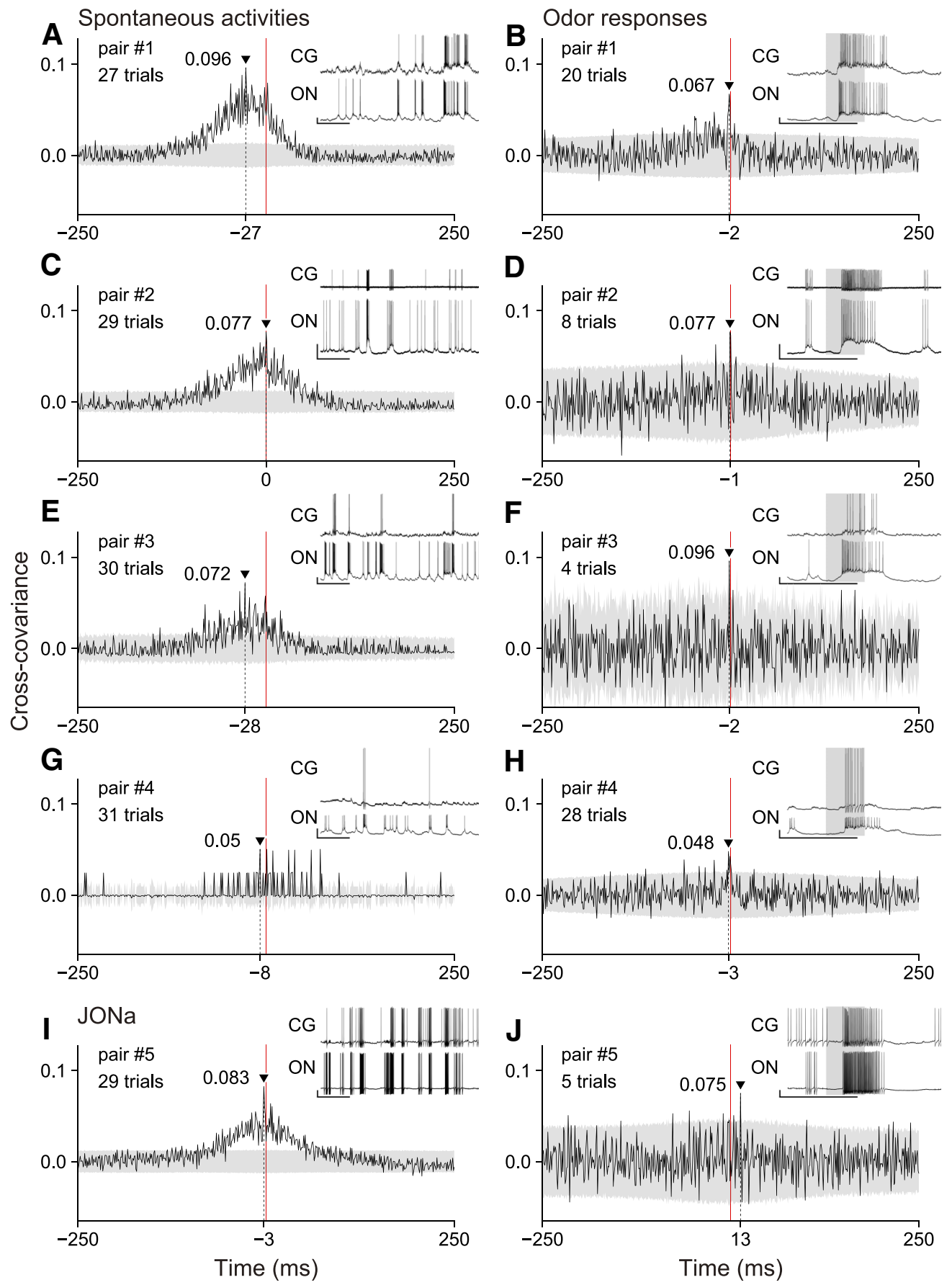

Figure 5. Cross-covariograms of spike activities of four pairs of a Type $1(\mathrm{C}$ and an MLONt $(\boldsymbol{A}-\boldsymbol{H})$ and a pair of a Type 1 (G and J0Na (I, $\boldsymbol{J})$. Cross-Covariance is plotted against the time lag from spike activities of a Type 1 CG to those of an MBON during spontaneous activities $(\boldsymbol{A}, \boldsymbol{C}, \boldsymbol{E}, \boldsymbol{G}, \boldsymbol{I})$ and odor responses $(\boldsymbol{B}, \boldsymbol{D}, \boldsymbol{F}, \boldsymbol{H}, \boldsymbol{J})$. Negative and positive time lags mean spike activities of an MBON precede and follow those of a Type 1 CG, respectively. The peak value and its position are indicated in each cross-covariogram. Gray area represents the mean \pm 2 SD range. Red vertical line indicates the origin (zero point) of the time lag. Insets, Part of neural activity recordings used for analysis. Top, CG. Bottom, MBON. Gray rectangles in the insets represent a $0.5 \mathrm{~s}$ period from the arrival of odor-prone air to the antenna, estimated using a photo-ionization detector. Calibration: Insets, $10 \mathrm{mV}$ (vertical), $1 \mathrm{~s}$ (horizontal). 0N, MB output neuron.

cross-correlation, in which the shuffle corrector allows the effect of firing rate changes induced by external sensory stimuli to be removed (see Cross-covariance analysis of spike activities of a Type 1 CG and an MBON) (Brody, 1999).

We analyzed four of eight pairs of a Type 1 CG and an MLONt in which continuous 25-30 s spontaneous spike activities and odor response spike activities were recorded. In three pairs of a Type 1 CG and an MLONt, cross-covariograms of the spike activities had a broad positive peak when no odor stimuli were applied (Fig. 5A,C,E, pairs 1-3). When spike activities of an
MLONt were shifted by -80 to $30 \mathrm{~ms}$ from those of a Type $1 \mathrm{CG}$, the cross-covariance exceeded a significance level (2 times of SD) and had a maximum value at a time lag of $-27,0$, or $-28 \mathrm{~ms}$. Cross-covariograms during odor responses had an extremely sharp positive peak, which exceeded the significance level by -4 to $0 \mathrm{~ms}$ and had a maximum value at -2 or $-1 \mathrm{~ms}$ (Fig. $5 B, D, F)$. The sharp peak of cross-covariance with a short time lag suggests that spikes of the Type 1 CG are, at least in part, produced by spikes of the MLONt with a short latency. We noticed that the broad peak seen during spontaneous activities was also seen during 
Spontaneous activities

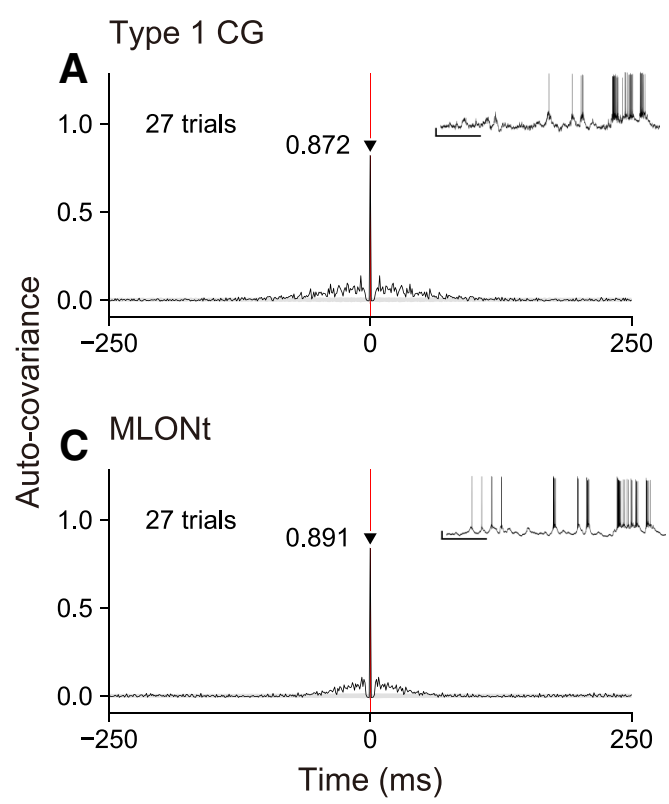

Odor responses

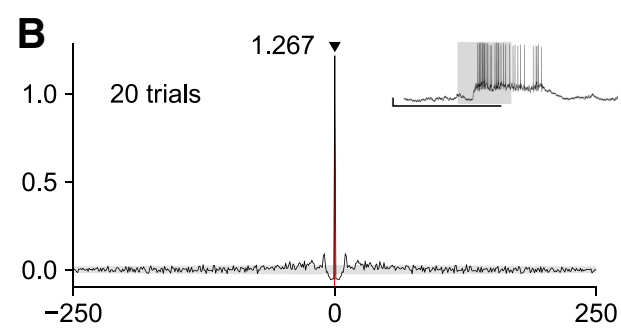

D

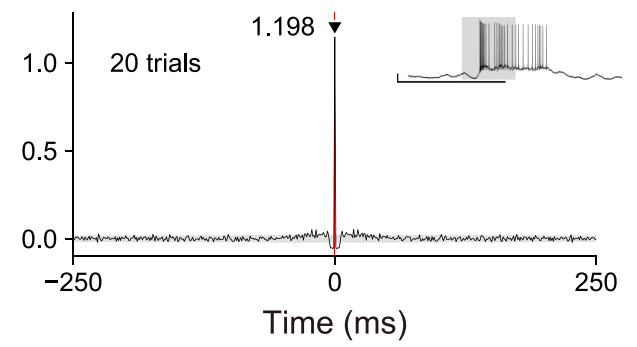

Figure 6. Typical examples of auto-covariograms of spike activities of a Type $1 \mathrm{CG}(\boldsymbol{A}, \boldsymbol{B})$ and an $\operatorname{MLONt}(\boldsymbol{C}, \boldsymbol{D})$ in pair 1. Covariograms and insets are as in Figure 5.

odor responses, although the magnitude was much smaller. The smaller amplitude of the broad cross-covariance peak obviously reflects higher firing rates of these neurons during odor responses; we divided $C(\tau)$ by the geometric mean firing rates for calculation of $\operatorname{COV}(\tau)$ (see Cross-covariance analysis of spike activities of a Type 1 $\mathrm{CG}$ and an $\mathrm{MBON}$ ).

In another pair (pair 4), the Type 1 CG fired less frequently during spontaneous activities than a typical Type 1 CG (Fig. $5 G$, inset). Its excitatory responses to odor stimuli consisted of spikes of $<10$ (Fig. 5I, inset). Infrequent spike bursts and occasional solitary spikes of the Type 1 CG, however, seemed to coincide with those of the MLONt. During spontaneous activities, cross-covariance of the binary spike sequence occasionally exceeded a significance level at -30 to $30 \mathrm{~ms}$, but a broad peak observed in pairs 1-3 was absent. This may be partly because the data consisted of very few spikes; thus, calculation of cross-covariance based on binary spike sequences was less effective. During odor responses, the Type 1 CG produced a few more spikes than those produced during spontaneous activities, and the cross-covariogram had a sharp peak with a short $(-3 \mathrm{~ms})$ time lag as in pairs $1-3$.

JONa, another type of MBON (Fig. 2G), was recorded simultaneously with the Type 1 CG (pair 5). As in the case of the MLONt, JONa exhibited spontaneous burst activities and excitatory responses to odor stimuli with transient increases in firing rate (Fig. 5I, J, insets). The cross-covariogram during spontaneous activities had a broad peak around the origin (zero time lag point) similar to that in pairs of the Type 1 CG and the MLONt (Fig. 5E). During odor responses, however, the cross-covariogram had a sharp peak at $13 \mathrm{~ms}$, distant from the origin (Fig. $5 \mathrm{~J}$ ). The significance of the positive value of the time lag was unclear. No sharp cross-covariance peak near the origin was found in this pair.

Finally, during the course of this study, we found no evidence of oscillatory activities of MBONs or CGs; namely, we observed no periodic peaks in auto-covariograms of a Type 1 CG and an MLONt during odor responses or during sponta- neous activities. Auto-covariograms of the Type $1 \mathrm{CG}$ and the MLONt in pair 1 had a sharp peak $(>0.8)$ at the zero point independently of the presence or absence of odor stimuli, but they exhibited no periodic covariance peaks (Fig. 6). Similarly, periodic auto-covariance peaks were not detectable in any other pairs of a Type $1 \mathrm{CG}$ and an MLONt (data not shown).

\section{Discussion}

\section{CGs comprise feedback circuits and control the gain of odor responses of $\mathrm{MBONs}$}

We obtained unequivocal evidence that CGs comprise feedback pathways in the cockroach MB. Spikes of the Type 1 CG hyperpolarized the MLONt during odor stimulation, whereas those of the MLONt depolarized the Type 1 CG (Figs. 3, 4). Hyperpolarization of the MLONt during odor stimulation is best accounted for by reduction of spike activities of KCs. Indeed, it has been reported that KCs of the cockroach receive GABAergic inhibitory synaptic input, and this contributes to reduction in the number of their spikes during odor responses (Demmer and Kloppenburg, 2009). Direct synaptic connections from the MLONt to the Type 1 CG are suggested by a short latency ( $<2 \mathrm{~ms}$ ) of EPSPs in the CG following spikes of the MLONt (Fig. 4) and are supported by overlaps between axon terminals of the MLONt and dendrites of the Type 1 CG (Fig. 2E,F). A short time lag (1-3 ms) of the sharp crosscovariance peak of spike activities of these neurons during odor responses (Fig. 5) strengthens this suggestion.

Notably, organizations of feedback circuits in the MB of cockroaches differ from those reported in other insect species. CGs receive synapses from $\mathrm{MBONs}$ in the protocerebrum, whereas GABAergic feedback neurons of the MB of other insect species receive synapses from KCs in the lobes, which may allow control of the gain of odor responses of KCs over a wide range of stimulus intensities (Papadopoulou et al., 2011; Lin et al., 2014). Because MBONs play critical roles in the control of behavioral responses of learned odors (in fruit-flies) (Plaçais et al., 2013; Owald et al., 2015), feedback from MBONs to KCs 


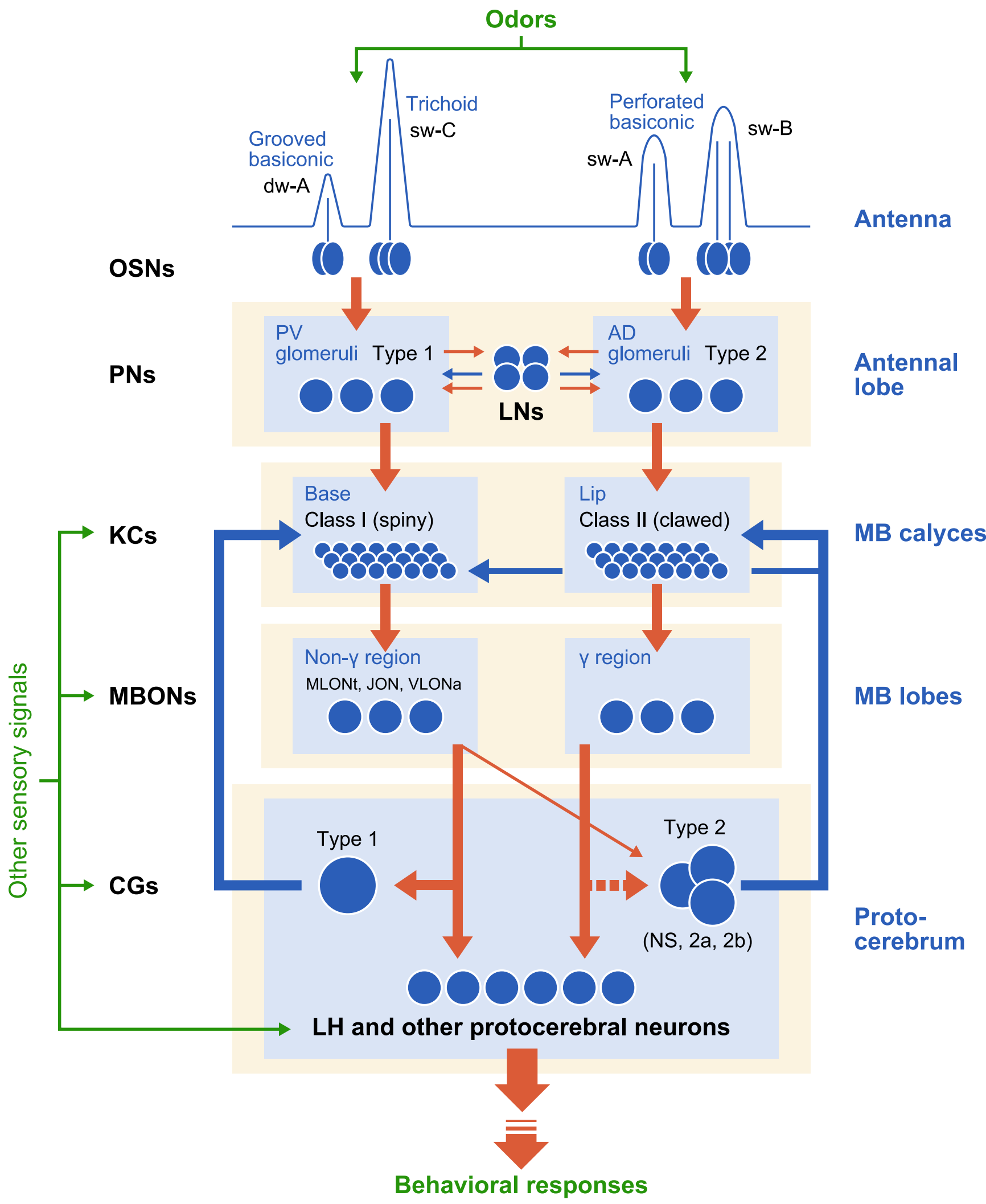

Figure 7. Separate but interactive olfactory processing streams from the periphery to the MB proposed for the cockroach MB. Blue and red pathways represent parallel processing streams, comprising Type 1 and Type 2 PNs, Class I and Class II KCS, separate classes of MBONs (with dendrites in non- $\gamma$ and $\gamma$ areas of the lobes), and CGs (Type 1 and Type 2), respectively. Circles on the pathways represent cell bodies of neurons, showing that the number of KCs is much larger than that of neurons in other processing stages. Data were not available for MBONs with dendrites in the $\gamma$ area, the termination area of Class II KCs. Dashed line indicates evidence for the pathway is not yet convincing. Many neurons of the MB and the protocerebrum exhibit responses to nonolfactory sensory signals (Mizunami et al., 1998c; Li and Strausfeld, 1999; Nishino et al., 2012; Takahashi et al., 2017), indicating integration of olfactory and nonolfactory signals. The significance of parallel olfactory processing systems for the control of olfactory behavior remains to be investigated. 
may allow control of the gain of odor responses to match the requirement of odor tracking behavior in the natural environment, in which odors are distributed in a highly complicated manner (see Nishino et al., 2018).

\section{Cross-covariance of spikes between the Type 1 CG and the MLONt suggests synchronized spike activities of MBONs during odor responses}

In cross-covariance analysis of spike activities of the Type 1 CG and the MLONt, we consistently observed different shapes and locations of a cross-covariance peak during spontaneous activities and odor responses (Fig. 5). Cross-covariograms during spontaneous activities had a broad peak across a range of $\sim 100$ $\mathrm{ms}$ around the origin, which we consider to reflect coincidence of timing of spike burst activities between the two neurons rather than precise coincidence of timing of individual spikes. During odor responses, a broad peak of covariance was less prominent; and instead, there was a sharp peak with a time lag of -3 to -1 ms, meaning that the MLONt spikes precede the Type 1 CG spikes by 1-3 ms. The sharp peak most likely reflects coincident occurrence of individual spikes of an MLONt and a Type 1 CG.

Why was a sharp peak of cross-covariance of spike activities of the Type 1 CG and the MLONt, which should reflect the occurrence of some spikes of the Type 1 CG in close coincidence with spikes of the MLONt, found during odor responses but not during spontaneous activities? The most plausible explanation is that because a CG receives only weak synaptic input from an individual MBON, a population of MBONs needs to coincidently spike for production of a spike in a CG during odor responses. Such synchronous spike firing of a population of MBONs during odor responses is most probably achieved by synchronous spike firings of populations of KCs, as is found in other species of insects. In locusts, a population of KCs exhibits synchronized spike firing during odor responses, which reflect synchronous spike firing of a population of PNs (Perez-Orive et al., 2002, 2004; Jortner et al., 2007). Synchronous spikes were also found among multiple ML (or $\beta$-lobe) output neurons (bLNs) during odor responses, whereas the synchronicity was less during spontaneous spike activities (Cassenaer and Laurent, 2007, 2012).

Interestingly, we found no evidence of oscillatory spike activities in the Type 1 CG or the MLONt in auto-covariance analysis during spontaneous activities or during odor responses (Fig. 6). This is in contrast to findings in locusts, in which periodic oscillation of membrane potential has been observed in all types of olfactory neurons, namely, in PNs, KCs, MBONs, and a GABAergic feedback neuron. In cockroaches, synchronous spikes among populations of olfactory neurons may be produced without oscillatory spike activities, although studies are needed to determine whether other types of olfactory neurons, such as PNs and KCs, exhibit no synchronous spikes.

\section{Interactive parallel odor processing streams with recurrent inhibitory pathways in the MB of cockroaches}

As illustrated in Figure 1, Type 1 and Type 2 PNs receive signals from different classes of OSNs housed in different types of sensilla on antennae (Watanabe et al., 2017) and terminate in base and lip regions of the MB calyces, in which Class I and Class II KCs extend dendrites, respectively (Nishino et al., 2018). The base and lip regions receive dense terminal arborizations of Type 1 and Type 2 CGs, respectively, with a part of the base region also receiving sparse terminal arborizations of Type 2 CGs (Takahashi et al., 2017).
What olfactory signals are conveyed by Type 1 and Type 2 PNs and then processed in a partially segregated manner in the $\mathrm{MB}$ circuit remain to be characterized. It has been argued that Type 1 PNs exhibit higher odor specificities than those of Type 2 PNs and that temporal activity of individual Type $1 \mathrm{PNs}$ is more suited for encoding odor-specific information (Watanabe et al., 2017). On the other hand, Type 2 PNs exhibit on-phasic early or late responses to odor stimulation, and the early responses might be suitable for coding the timing and concentration of odor stimuli (Watanabe et al., 2017). These distinct features might be somewhat analogous to the distinction between PNs in a medial antennal lobe tract (m-ALT, referred to also as the medial antenno-protocerebral tract [m-APT]) and those in the lateral antennal lobe tract (1-ALT, referred to as the l-APT) in honey bees (Galizia and Rössler, 2010; Schmuker et al., 2011; Brill et al., 2013; Carcaud et al., 2018).

In Figure 7, we propose a schematic diagram of separate but interactive parallel odor processing streams in cockroaches. A Type 1 CG, the termination areas of which exactly match the dendritic area of Class I KCs, inhibited spike activities of the MLONt and JONa, for which dendritic areas (non- $\gamma$ area) are termination areas of Class I KCs. We also obtained evidence that the MLONt make synapses with the Type 1 CG. Therefore, there is a recurrent inhibitory pathway comprising Class I KCs, MBONs with dendrites in the non- $\gamma$ area, and the Type 1 CG. In contrast, our knowledge of feedback circuits that process information of Class II KCs is still incomplete. We observed that the NS CG, the termination areas of which profusely cover the dendritic areas of Class II KCs but sparsely cover a part of the dendritic areas of Class I KCs (Takahashi et al., 2017), strongly inhibits spike activities of VLONa (Fig. $3 K$ ) with its effect on the MLONt being very weak (Fig. 3I). This matches the observations that the NS CG had dendrites in proximity to terminal branches of VLONa (Fig. 2T) but in much less proximity to those of MLONt (Fig. $2 J, N)$. Since dendrites of VLONa were observed in non- $\gamma$ areas but not in the $\gamma$ area, it seems unlikely that VLONa comprises a feedback pathway involving Class II KCs. Therefore, although there are parallel processing streams from different types of OSNs housed in different sensilla on antennae to Class I and Class II KCs in the MB, via Type 1 and Type 2 PNs, interactions between the two streams are evident at the level of feedback pathways in the MB, which matches the "separate but interactive" parallel olfactory pathways discussed by Galizia and Rössler (2010). Unfortunately, we did not record from MBONs with their dendrites mainly in the $\gamma$ area in this study; thus, more studies are needed to better characterize organizations of $\mathrm{MB}$ output pathways. On account of such a limitation, our study is the first to suggest that the higher-olfactory center is organized into separate but interactive processing streams that deal with signals from different types of OSNs.

It should be pointed out that integrations of signals from different streams occur not only in the MB feedback pathway but also in an earlier olfactory processing stage in cockroaches, namely, local interneurons in the antennal lobe integrate signals from both streams, and this results in overlapping representations of odors by Type 1 and Type 2 PNs (Watanabe et al., 2017). Since cockroaches have been used as model animals to study innate and learned olfactory behavior (Bell and Tobin, 1981; Lockey and Willis, 2015; Hosono et al., 2016), they should provide pertinent models for investigating the significance of parallel olfactory processing systems in terms of the control of innate and learned olfactory behavior. 


\section{References}

Aarts E, Verhage M, Veenvliet JV, Dolan CV, van der Sluis S (2014) A solution to dependency: using multilevel analysis to accommodate nested data. Nat Neurosci 17:491-496.

Bair W, Zohary E, Newsome WT (2001) Correlated firing in macaque visual area MT: time scales and relationship to behavior. J Neurosci 21:16761697.

Bell W, Tobin T (1981) Orientation to sex pheromone in the American cockroach: analysis of chemo-orientation mechanisms. J Insect Physiol 27:501-508.

Brill MF, Rosenbaum T, Reus I, Kleineidam CJ, Nawrot MP, Rössler W (2013) Parallel processing via a dual olfactory pathway in the honeybee. J Neurosci 33:2443-2456.

Brody CD (1999) Correlations without synchrony. Neural Comput 11: 1537-1551.

Buhl E, Roberts A, Soffe SR (2012) The role of a trigeminal sensory nucleus in the initiation of locomotion. J Physiol 590:2453-2469.

Carcaud J, Giurfa M, Sandoz JC (2018) Differential processing by two olfactory subsystems in the honeybee brain. Neuroscience 374:33-48.

Cassenaer S, Laurent G (2007) Hebbian STDP in mushroom bodies facilitates the synchronous flow of olfactory information in locusts. Nature 448:709-713.

Cassenaer S, Laurent G (2012) Conditional modulation of spike-timingdependent plasticity for olfactory learning. Nature 482:47-52.

Demmer H, Kloppenburg P (2009) Intrinsic membrane properties and inhibitory synaptic input of Kenyon cells as mechanisms for sparse coding? J Neurophysiol 102:1538-1550.

Faraway JJ (2006) Extending the linear model with R: generalized linear, mixed effects and nonparametric regression models. Boca Raton, FL: Chapman and Hall/CRC.

Fujimura K, Yokohari F, Tateda H (1991) Classification of antennal olfactory receptors of the cockroach, Periplaneta americana L. Zool Sci $8: 243-255$.

Galizia CG, Rössler W (2010) Parallel olfactory systems in insects: anatomy and function. Annu Rev Entomol 55:399-420.

Gupta N, Stopfer M (2014) A temporal channel for information in sparse sensory coding. Curr Biol 24:2247-2256.

Hosono S, Matsumoto Y, Mizunami M (2016) Interaction of inhibitory and facilitatory effects of conditioning trials on long-term memory formation. Learn Mem 23:669-678.

Jortner RA, Farivar SS, Laurent G (2007) A simple connectivity scheme for sparse coding in an olfactory system. J Neurosci 27:1659-1669.

Li Y, Strausfeld NJ (1999) Multimodal efferent and recurrent neurons in the medial lobes of cockroach mushroom bodies. J Comp Neurol 409: 647-663.

Lin AC, Bygrave AM, de Calignon A, Lee T, Miesenböck G (2014) Sparse, decorrelated odor coding in the mushroom body enhances learned odor discrimination. Nat Neurosci 17:559-568.

Liu C, Plaçais PY, Yamagata N, Pfeiffer BD, Aso Y, Friedrich AB, Siwanowicz I, Rubin GM, Preat T, Tanimoto H (2012) A subset of dopamine neurons signals reward for odour memory in Drosophila. Nature 488:512516.

Lockey JK, Willis MA (2015) One antenna, two antennae, big antennae, small: total antennae length, not bilateral symmetry, predicts odortracking performance in the American cockroach Periplaneta americana. J Exp Biol 218:2156-2165.

Menzel R, Giurfa M (2006) Dimensions of cognition in an insect, the honeybee. Behav Cogn Neurosci Rev 5:24-40.

Mizunami M, Iwasaki M, Okada R, Nishikawa M (1998a) Topography of modular subunits in the mushroom bodies of the cockroach. J Comp Neurol 399:153-161.

Mizunami M, Iwasaki M, Okada R, Nishikawa M (1998b) Topography of four classes of Kenyon cells in the mushroom bodies of the cockroach. J Comp Neurol 399:162-175.

Mizunami M, Okada R, Li Y, Strausfeld NJ (1998c) Mushroom bodies of the cockroach: activity and identities of neurons recorded in freely moving animals. J Comp Neurol 402:501-519.

Nishino H, Mizunami M (1998) Giant input neurons of the mushroom body: intracellular recording and staining in the cockroach. Neurosci Lett 246:57-60.

Nishino H, Yamashita S, Yamazaki Y, Nishikawa M, Yokohari F, Mizunami M (2003) Projection neurons originating from thermo- and hygrosensory glomeruli in the antennal lobe of the cockroach. J Comp Neurol 455:40-55.

Nishino H, Iwasaki M, Yasuyama K, Hongo H, Watanabe H, Mizunami M (2012) Visual and olfactory input segregation in the mushroom body calyces in a basal neopteran, the American cockroach. Arthropod Struct Dev 41:3-16.

Nishino H, Iwasaki M, Paoli M, Kamimura I, Yoritsune A, Mizunami M (2018) Spatial receptive fields for odor localization. Curr Biol 28:600608.e3.

Owald D, Felsenberg J, Talbot CB, Das G, Perisse E, Huetteroth W, Waddell S (2015) Activity of defined mushroom body output neurons underlies learned olfactory behavior in Drosophila. Neuron 86:417-427.

Pang JJ, Yang Z, Jacoby RA, Wu SM (2018) Cone synapses in mammalian retinal rod bipolar cells. J Comp Neurol 526:1896-1909.

Papadopoulou M, Cassenaer S, Nowotny T, Laurent G (2011) Normalization for sparse encoding of odors by a wide-field interneuron. Science 332:721-725.

Perez-Orive J, Mazor O, Turner GC, Cassenaer S, Wilson RI, Laurent G (2002) Oscillations and sparsening of odor representations in the mushroom body. Science 297:359-365.

Perez-Orive J, Bazhenov M, Laurent G (2004) Intrinsic and circuit properties favor coincidence detection for decoding oscillatory input. J Neurosci 24:6037-6047.

Plaçais PY, Trannoy S, Friedrich AB, Tanimoto H, Preat T (2013) Two pairs of mushroom body efferent neurons are required for appetitive long-term memory retrieval in Drosophila. Cell Rep 5:769-780.

Schmuker M, Yamagata N, Nawrot MP, Menzel R (2011) Parallel representation of stimulus identity and intensity in a dual pathway model inspired by the olfactory system of the honeybee. Front Neuroeng 4:17.

Stopfer M (2014) Central processing in the mushroom bodies. Curr Opin Insect Sci 6:99-103.

Strausfeld NJ, Li Y (1999a) Organization of olfactory and multimodal afferent neurons supplying the calyx and pedunculus of the cockroach mushroom bodies. J Comp Neurol 409:603-625.

Strausfeld NJ, Li Y (1999b) Representation of the calyces in the medial and vertical lobes of cockroach mushroom bodies. J Comp Neurol 409: $626-646$.

Szyszka P, Ditzen M, Galkin A, Galizia CG, Menzel R (2005) Sparsening and temporal sharpening of olfactory representations in the honeybee mushroom bodies. J Neurophysiol 94:3303-3313.

Takahashi N, Katoh K, Watanabe H, Nakayama Y, Iwasaki M, Mizunami M, Nishino H (2017) Complete identification of four giant interneurons supplying mushroom body calyces in the cockroach Periplaneta americana. J Comp Neurol 525:204-230.

Watanabe H, Haupt SS, Nishino H, Nishikawa M, Yokohari F (2012) Sensillum-specific, topographic projection patterns of olfactory receptor neurons in the antennal lobe of the cockroach Periplaneta americana. J Comp Neurol 520:1687-1701.

Watanabe H, Nishino H, Mizunami M, Yokohari F (2017) Two parallel olfactory pathways for processing general odors in a cockroach. Front Neural Circuits 11:32.

Yamazaki Y, Nishikawa M, Mizunami M (1998) Three classes of GABA-like immunoreactive neurons in the mushroom body of the cockroach. Brain Res 788:80-86. 